\title{
Direct and inverse spin-orbit torques
}

\author{
Frank Freimuth* Stefan Blügel, and Yuriy Mokrousov \\ Peter Grünberg Institut and Institute for Advanced Simulation, \\ Forschungszentrum Jülich and JARA, 52425 Jülich, Germany
}

(Dated: September 20, 2018)

\begin{abstract}
In collinear magnets lacking inversion symmetry application of electric currents induces torques on the magnetization and conversely magnetization dynamics induces electric currents. The two effects, which both rely on spin-orbit interaction (SOI), are reciprocal to each other and denoted direct spinorbit torque (SOT) and inverse spin-orbit torque (ISOT), respectively. We derive expressions for SOT and ISOT within the Kubo linear response formalism. We show that expressions suitable for density-functional theory calculations can be derived either starting from a Kohn-Sham Hamiltonian with time-dependent exchange field or by expressing general susceptibilities in terms of the KohnSham susceptibilities. For the case of magnetic bilayer systems we derive the general form of the ISOT current induced under ferromagnetic resonance. Using ab initio calculations within densityfunctional theory we investigate SOT and ISOT in $\mathrm{Co} / \mathrm{Pt}(111)$ magnetic bilayers. We determine the spatial distribution of spin and charge currents as well as torques in order to expose the mechanisms underlying SOT and ISOT and to highlight their reciprocity on the microscopic level. We find that the spin Hall effect is position-dependent close to interfaces.

PACS numbers: 72.25.Ba, 72.25.Mk, 71.70.Ej, 75.70.Tj
\end{abstract}

\section{INTRODUCTION}

In ferromagnetic materials Faraday's law of induction needs to be generalized to include so-called spinmotive forces, i.e., electric fields induced by the magnetization dynamics [1 3]. The spinmotive force can be interpreted as the reciprocal of the current-induced torque: A moving domain wall induces a spinmotive force and conversely an applied current drives domain wall motion. Thus, the electric fields induced by magnetization dynamics generate a feedback effect on the magnetization via the current-induced torques which they produce [4].

Spinmotive forces do not only occur in noncollinear magnetic structures such as domain-walls [5] and skyrmions [6] but can arise also in collinear magnets due to the interplay of spin orbit interaction (SOI) with bulk or structural inversion asymmetry [7, 8]. Spin-orbit torques (SOTs) [9 17], i.e., current-induced torques originating from SOI in inversion asymmetric collinear magnets, are the reciprocal to the electric fields induced by magnetization dynamics in collinear magnets [18, 19]. Thus, we will denote the latter as inverse spin-orbit torques (ISOTs) in the following. ISOTs constitute a special case of spinmotive forces.

While earlier experiments on SOTs estimated the current-induced torques indirectly from the onset of nucleation of reversed domains [20] or magnetization switching at critical current densities [21 23] direct measurements of SOTs have been performed recently in bilayer systems and the SOT has been determined as a function of magnetization direction $\hat{M}$ [24 26]. Two qualitatively different SOT components are found in these experiments on bilayer systems, the first one is an even function of $\hat{\boldsymbol{M}}$, the second one is an odd function. Denoting the applied in-plane electric field by $\boldsymbol{E}$ and the unit vector in the out-of-plane direction by $\hat{\boldsymbol{e}}_{z}$, they are given by $\boldsymbol{T}^{\text {even }}=T^{\text {even }} \hat{\boldsymbol{M}} \times\left[\left(\hat{\boldsymbol{e}}_{z} \times \boldsymbol{E}\right) \times \hat{\boldsymbol{M}}\right]$ and $\boldsymbol{T}^{\text {odd }}=T^{\text {odd }}\left(\hat{\boldsymbol{e}}_{z} \times \boldsymbol{E}\right) \times \hat{\boldsymbol{M}}$ to lowest order in $\hat{\boldsymbol{M}}$.

In bilayer systems based on $5 \mathrm{~d}$ transition metals with large spin Hall effect (SHE), such as $\mathrm{AlO}_{x} / \mathrm{Co} / \mathrm{Pt}$, $\mathrm{MgO} / \mathrm{CoFeB} / \mathrm{Ta}$ and $\mathrm{CoFeB} / \mathrm{W}$, the dominant contribution to $\boldsymbol{T}^{\text {even }}$ arises from the SHE [22, 23, 27 31]. Conversely, in $\mathrm{Ni}_{80} \mathrm{Fe}_{20} / \mathrm{Pt}$ the spin current pumped into $\mathrm{Pt}$ by exciting the ferromagnetic resonance (FMR) of $\mathrm{Ni}_{80} \mathrm{Fe}_{20}$ induces an electric field via the inverse spin Hall effect (ISHE) 32 34. Rashba SOI provides an important contribution to $\boldsymbol{T}^{\text {odd }}$ in these bilayer systems [14, 15]. Due to the reciprocity between SOT and ISOT, an additional ISOT is expected as well from the Rashba SOI at the bilayer interface [7, 8]. This theoretical prediction, that the ISOT in bilayer systems should not arise purely from the combination of spin pumping and ISHE, is supported by the experimental observation that for the reciprocal phenomenon, the SOT, $\boldsymbol{T}^{\text {odd }}$ can be as large as or even larger than $\boldsymbol{T}^{\text {even }}$ [24 26].

So far only the dc voltage due to FMR-driven ISOT has been studied intensively in bilayer systems [34 38]. However, after the theoretical prediction [39] that the ac component is expected to be much larger than the dc one, several recent experiments have been devoted to its measurement [40 42]. As will be discussed in this work it is expected from the reciprocity of ISOT and SOT that the dc voltage generated by the FMR-driven ISOT is proportional to $\boldsymbol{T}^{\text {even }}$, while the ac voltage is determined by both $\boldsymbol{T}^{\text {even }}$ and $\boldsymbol{T}^{\text {odd }}$. Since the ac voltages associated with $\boldsymbol{T}^{\text {even }}$ and $\boldsymbol{T}^{\text {odd }}$ exhibit a phase difference of $\pm 90^{\circ}$ a non-trivial phase relationship between ac signal and magnetization trajectory is expected. Phasesensitive measurements of the ac ISOT-signal induced under FMR can thus be complementary to experiments 
on the SOT phenomenon. Both types of experiments, i.e., measuring the induced voltage under FMR on the one hand and measuring on the other hand the currentinduced torque on the magnetization, can thus serve to determine $\boldsymbol{T}^{\text {even }}$ and $\boldsymbol{T}^{\text {odd }}$ and from them the parameters needed to model them, notably spin-diffusion length, spin-mixing conductance, SHE-angle as well as Rashba and Dresselhaus parameters.

This article is organized as follows: In Sec. II we discuss the Kubo formalism expressions for both SOT and ISOT. In the case of the SOT phenomenon, the torque on the magnetization is given by $\boldsymbol{T}=\boldsymbol{t} \boldsymbol{E}$, which defines the torkance tensor $\boldsymbol{t}$. We show that also the ISOT can be captured conveniently in terms of $\boldsymbol{t}$, which is a consequence of the reciprocity between SOT and ISOT. In Sec. IIB we show that expressions for both ISOT and Gilbert damping can be derived consistently based on Kohn-Sham theory with a time-dependent exchange field. In Sec. IIC we show that these expressions can also be obtained by expressing general many-body susceptibilities in terms of the corresponding Kohn-Sham susceptibilities. Exploiting the reciprocity between SOT and ISOT we then predict in Sec. III the angular dependence of ISOT in magnetic bilayers from the angular dependence of SOT recently measured in these systems. In particular we derive and discuss the FMR-induced currents for various magnetization directions in bilayer systems. In Sec. IIIB a minimal model to describe even SOT and ISOT in bilayers is discussed. In Sec. III C we consider odd SOT and ISOT within the Boltzmann formalism. In Sec. IV] we investigate SOT and ISOT for a magnetic bilayer composed of a Co layer on Pt(111). Computing spin currents, ISOT-induced charge currents and torkances layer-resolved we make contact with phenomenological models and extract model parameters. We conclude by a summary in Sec. V.

\section{RELATIONSHIP BETWEEN DIRECT SOT AND INVERSE SOT}

\section{A. Induced currents under time-dependent magnetization}

Reciprocity between current-induced torques and spinmotive forces has been discussed in detail in the framework of phenomenological modelling [18, 19, 43, 44]. In this section, we revisit this reciprocity on the basis of the Kubo linear response formalism, which is well-suited to study SOT and ISOT from first principles.

Within the local spin density approximation (LSDA) the interacting many-electron system is described by an effective single-particle Hamiltonian of the form

$$
H(\boldsymbol{r}, t)=H_{0}(\boldsymbol{r})-\boldsymbol{m} \cdot \hat{\boldsymbol{M}}(t) \Omega^{\mathrm{xc}}(\boldsymbol{r}),
$$

where the time-independent $H_{0}$ contains kinetic energy, scalar potential and SOI, while the second term on the right-hand side describes the exchange interaction. $\hat{\boldsymbol{M}}(t)$ is a normalized vector which points in the direction of magnetization. In order to describe the electronic system at the ferromagnetic resonance we assume that $\hat{\boldsymbol{M}}(t)$ is precessing. The time-dependence of the Hamiltonian arises from this precession of magnetization. $\boldsymbol{m}=-\mu_{\mathrm{B}} \boldsymbol{\sigma}$ with the Bohr magneton $\mu_{\mathrm{B}}$ and the vector of Pauli spin matrices $\boldsymbol{\sigma}=\left(\sigma_{x}, \sigma_{y}, \sigma_{z}\right)^{\mathrm{T}}$ is the spin magnetic moment operator. $\Omega^{\mathrm{xc}}(\boldsymbol{r})$ is the exchange field, i.e., the difference between the potentials of majority and minority electrons $\Omega^{\mathrm{xc}}(\boldsymbol{r})=\frac{1}{2 \mu_{\mathrm{B}}}\left(V_{\text {minority }}^{\mathrm{eff}}(\boldsymbol{r})-V_{\text {majority }}^{\mathrm{eff}}(\boldsymbol{r})\right)$. Around the time $t$ we can approximate the motion of $\hat{M}$ by

$$
\hat{\boldsymbol{M}}(t+\Delta t)-\hat{\boldsymbol{M}}(t) \simeq \frac{d \hat{\boldsymbol{M}}(t)}{d t} \Delta t \simeq \frac{d \hat{\boldsymbol{M}}(t)}{d t} \frac{\sin (\omega \Delta t)}{\omega}
$$

for small time changes $\Delta t$ and a small but arbitrary frequency $\omega$ with $\omega \Delta t \ll 1$. Likewise, the Hamiltonian can be approximated as

$$
H(\boldsymbol{r}, t+\Delta t) \simeq H(\boldsymbol{r}, t)-\boldsymbol{m} \cdot \frac{d \hat{\boldsymbol{M}}(t)}{d t} \Omega^{\mathrm{xc}}(\boldsymbol{r}) \frac{\sin (\omega \Delta t)}{\omega} .
$$

The $\Delta t$-dependent term

$$
\begin{aligned}
& V(\boldsymbol{r}, \Delta t)=-\boldsymbol{m} \cdot \frac{d \hat{\boldsymbol{M}}(t)}{d t} \Omega^{\mathrm{xc}}(\boldsymbol{r}) \frac{\sin (\omega \Delta t)}{\omega} \\
& =-\boldsymbol{m} \cdot\left[\hat{\boldsymbol{M}}(t) \times\left(\frac{d \hat{\boldsymbol{M}}(t)}{d t} \times \hat{\boldsymbol{M}}(t)\right)\right] \Omega^{\mathrm{xc}}(\boldsymbol{r}) \frac{\sin (\omega \Delta t)}{\omega} \\
& =\frac{\sin (\omega \Delta t)}{\omega}\left(\hat{\boldsymbol{M}}(t) \times \frac{d \hat{\boldsymbol{M}}(t)}{d t}\right) \cdot \boldsymbol{T}(\boldsymbol{r}, t)
\end{aligned}
$$

acts as a time-dependent perturbation on the eigenstates of $H(\boldsymbol{r}, t)$. Here, $\mathcal{T}(\boldsymbol{r}, t)=\boldsymbol{m} \times \hat{\boldsymbol{M}}(t) \Omega^{\mathrm{xc}}(\boldsymbol{r})$ is the torque operator.

Within linear response the current density in $\alpha$ direction, $j_{\alpha}$, induced by the time-dependent perturbation Eq. (4) is given by

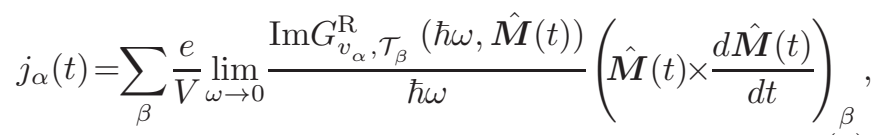

where $e>0$ is the elementary positive charge, $V$ is the volume and $G_{v_{\alpha}}^{\mathrm{R}}, \mathcal{T}_{\beta}(\hbar \omega, \hat{\boldsymbol{M}})$ is the Fourier transform of the retarded velocity-torque correlation function, i.e.,

$$
G_{v_{\alpha}, \mathcal{T}_{\beta}}^{\mathrm{R}}(\hbar \omega, \hat{\boldsymbol{M}})=-i \int_{0}^{\infty} d t e^{i \omega t}\left\langle\left[v_{\alpha}(t), \mathcal{T}_{\beta}(0)\right]_{-}\right\rangle,
$$

evaluated for the time-independent Hamiltonian

$$
H_{\hat{M}}(\boldsymbol{r})=H_{0}(\boldsymbol{r})-\boldsymbol{m} \cdot \hat{M} \Omega^{\mathrm{xc}}(\boldsymbol{r})
$$

of a system with magnetization in direction $\hat{M}=\hat{M}(t)$. Eq. (6) describes the correlation between the polar vector 
$\boldsymbol{v}$ and the axial vector $\mathcal{T}$. This polar-axial correlation is nonzero only when inversion symmetry is broken.

Next, we compare Eq. (5) to the expressions describing SOTs. Within linear response to an applied electric field $\boldsymbol{E}$ the SOT on the magnetization is $\boldsymbol{T}(\hat{\boldsymbol{M}})=\boldsymbol{t}(\hat{\boldsymbol{M}}) \boldsymbol{E}$, where the torkance tensor $\boldsymbol{t}(\hat{\boldsymbol{M}})$ is given by 31, 45]

$$
t_{\alpha \beta}(\hat{\boldsymbol{M}})=-e \lim _{\omega \rightarrow 0} \frac{\operatorname{Im} G_{\mathcal{T}_{\alpha}, v_{\beta}}^{\mathrm{R}}(\hbar \omega, \hat{\boldsymbol{M}})}{\hbar \omega}
$$

in terms of the Fourier transform of the retarded torquevelocity correlation function

$$
G_{\mathcal{T}_{\alpha}, v_{\beta}}^{\mathrm{R}}(\hbar \omega, \hat{\boldsymbol{M}})=-i \int_{0}^{\infty} d t e^{i \omega t}\left\langle\left[\mathcal{T}_{\alpha}(t), v_{\beta}(0)\right]_{-}\right\rangle
$$

of the system with Hamiltonian Eq. (7).

The spectral densities of the Green functions defined in Eq. (6) and in Eq. (9) are given by

$$
\begin{aligned}
S_{v_{\alpha}, \mathcal{T}_{\beta}}\left(t, t^{\prime}, \hat{\boldsymbol{M}}\right) & =\frac{1}{2 \pi}\left\langle\left[v_{\alpha}(t), \mathcal{T}_{\beta}\left(t^{\prime}\right)\right]_{-}\right\rangle, \\
S_{\mathcal{T}_{\alpha}, v_{\beta}}\left(t, t^{\prime}, \hat{\boldsymbol{M}}\right) & =\frac{1}{2 \pi}\left\langle\left[\mathcal{T}_{\alpha}(t), v_{\beta}\left(t^{\prime}\right)\right]_{-}\right\rangle
\end{aligned}
$$

and their Fourier transforms satisfy the relations

$$
\begin{aligned}
S_{\mathcal{T}_{\alpha}, v_{\beta}}(\hbar \omega, \hat{\boldsymbol{M}}) & =\left[S_{v_{\beta}, \mathcal{T}_{\alpha}}(\hbar \omega, \hat{\boldsymbol{M}})\right]^{*}, \\
\operatorname{Re}\left[S_{v_{\beta}, \mathcal{T}_{\alpha}}(\hbar \omega,-\hat{\boldsymbol{M}})\right] & =-\operatorname{Re}\left[S_{v_{\beta}}, \mathcal{T}_{\alpha}(\hbar \omega, \hat{\boldsymbol{M}})\right], \\
\operatorname{Im}\left[S_{v_{\beta}, \mathcal{T}_{\alpha}}(\hbar \omega,-\hat{\boldsymbol{M}})\right] & =\operatorname{Im}\left[S_{v_{\beta}, \mathcal{T}_{\alpha}}(\hbar \omega, \hat{\boldsymbol{M}})\right],
\end{aligned}
$$

from which follows

$$
S_{\mathcal{T}_{\alpha}, v_{\beta}}(\hbar \omega, \hat{\boldsymbol{M}})=-S_{v_{\beta}, \mathcal{T}_{\alpha}}(\hbar \omega,-\hat{\boldsymbol{M}})
$$

and thus

$$
G_{\mathcal{T}_{\alpha}, v_{\beta}}^{\mathrm{R}}(\hbar \omega, \hat{M})=-G_{v_{\beta}, \mathcal{T}_{\alpha}}^{\mathrm{R}}(\hbar \omega,-\hat{\boldsymbol{M}}) .
$$

This identity allows us to rewrite the magnetizationdynamics induced current density, Eq. (5), in terms of the torkance tensor as

$$
j_{\alpha}(t)=\frac{1}{V} \sum_{\beta} t_{\beta \alpha}(-\hat{\boldsymbol{M}}(t))\left(\hat{\boldsymbol{M}}(t) \times \frac{d \hat{\boldsymbol{M}}(t)}{d t}\right)_{\beta} .
$$

Eq. (14) is the central result of this subsection. It shows that it is very convenient to discuss the ISOT in terms of the very same torkance tensor $\boldsymbol{t}$ as the SOT. We note in passing that the torque-velocity correlations, which the torkance measures, govern also the DzyaloshinskiiMoriya interaction [45, 46].

It is convenient to decompose the torkance tensor into two components that are even and odd with respect to magnetization reversal, respectively [31]: $\boldsymbol{t}(\hat{\boldsymbol{M}})=$ $\boldsymbol{t}^{\text {even }}(\hat{\boldsymbol{M}})+\boldsymbol{t}^{\text {odd }}(\hat{\boldsymbol{M}})$, where $\boldsymbol{t}^{\text {even }}(\hat{\boldsymbol{M}})=[\boldsymbol{t}(\hat{\boldsymbol{M}})+$
$\boldsymbol{t}(-\hat{\boldsymbol{M}})] / 2$ and $\boldsymbol{t}^{\text {odd }}(\hat{\boldsymbol{M}})=[\boldsymbol{t}(\hat{\boldsymbol{M}})-\boldsymbol{t}(-\hat{\boldsymbol{M}})] / 2$. Separating $j_{\alpha}$ into the components due to $\boldsymbol{t}^{\text {even }}(\hat{\boldsymbol{M}})$ and $\boldsymbol{t}^{\text {odd }}(\hat{\boldsymbol{M}})$ yields

$$
\begin{aligned}
& j_{\alpha}^{\text {even }}(t)=\frac{1}{V} \sum_{\beta} t_{\beta \alpha}^{\text {even }}(\hat{\boldsymbol{M}}(t))\left(\hat{\boldsymbol{M}}(t) \times \frac{d \hat{\boldsymbol{M}}(t)}{d t}\right)_{\beta}, \\
& j_{\alpha}^{\text {odd }}(t)=-\frac{1}{V} \sum_{\beta} t_{\beta \alpha}^{\text {odd }}(\hat{\boldsymbol{M}}(t))\left(\hat{\boldsymbol{M}}(t) \times \frac{d \hat{\boldsymbol{M}}(t)}{d t}\right)_{\beta} .
\end{aligned}
$$

\section{B. Completing the response matrix}

When the electronic system is perturbed due to the time-dependence of the exchange field direction a current density is induced according to Eq. (51). This induced electric current is not the only response of the electrons to this time dependent perturbation: Additionally, the torque $-V \boldsymbol{\Lambda}\left(\hat{\boldsymbol{M}} \times \frac{d \hat{M}}{d t}\right)$ acts on the magnetization, where

$$
\Lambda_{\alpha \beta}=-\frac{1}{V} \lim _{\omega \rightarrow 0} \frac{\operatorname{Im} G_{\mathcal{T}_{\alpha}}^{\mathrm{R}}, \mathcal{T}_{\beta}(\hbar \omega, \hat{M})}{\hbar \omega} .
$$

The sum of all torques on the magnetization has to be zero from the point of view of an observer that rotates together with the magnetization:

$$
0=\boldsymbol{t} \boldsymbol{E}-V \boldsymbol{\Lambda}\left(\hat{\boldsymbol{M}} \times \frac{d \hat{\boldsymbol{M}}}{d t}\right)+\mu_{0} M V \hat{\boldsymbol{M}} \times \boldsymbol{H}^{\mathrm{eff}} .
$$

Here, the first term on the right-hand side is the SOT. Torques such as the Gilbert damping torque, which are exerted on the magnetization due to the magnetization dynamics, are described by the second term. The third term summarizes torques due to external magnetic fields and due to magnetic anisotropy. $M$ in the third term is the magnetization, i.e., $M V$ is the magnetic moment. In the presence of SOTs, the extended Landau-LifshitzGilbert equation runs

$$
\frac{d \hat{\boldsymbol{M}}}{d t}=-|\gamma| \hat{\boldsymbol{M}} \times \boldsymbol{H}^{\mathrm{eff}}+\boldsymbol{\alpha} \hat{\boldsymbol{M}} \times \frac{d \hat{\boldsymbol{M}}}{d t}-\frac{|\gamma| \boldsymbol{t} \boldsymbol{E}}{\mu_{0} M V}
$$

where $\gamma=g \mu_{0} \mu_{\mathrm{B}} / \hbar$ is the gyromagnetic ratio and $\boldsymbol{\alpha}$ is the Gilbert damping tensor. Comparison of Eq. (18) and Eq. (17) leads to

$$
\frac{1}{\gamma}=\frac{1}{2 \mu_{0} M} \sum_{\alpha \beta \delta} \epsilon_{\alpha \beta \delta} \Lambda_{\alpha \beta}^{\text {odd }} \hat{M}_{\delta}
$$

where $\epsilon_{\alpha \beta \delta}$ is the Levi-Civita symbol, and

$$
\boldsymbol{\alpha}=\frac{|\gamma| \boldsymbol{\Lambda}^{\text {even }}}{M \mu_{0}}
$$


It is straightforward to show that Eq. 20. combined with Eq. (16) reproduces the Gilbert damping expressions used within $a b$ initio calculations [47]. In the absence of SOI it is found that [48]

$$
\Lambda_{\alpha \beta}^{\mathrm{odd}}=-\frac{\hbar}{2 \mu_{\mathrm{B}}} \sum_{\gamma} \epsilon_{\alpha \beta \gamma} M_{\gamma}
$$

Inserting this result into Eq. (19) leads to the expected nonrelativistic value of $\gamma=-\frac{2 \mu_{0} \mu_{\mathrm{B}}}{\hbar}$ and $g=-2$.

If we consider the coupled problem where both the electric field and the magnetization dynamics drive both the electric current and induce torques, the even torkance $\boldsymbol{t}^{\text {even }}$ determines the off-diagonal elements of the symmetric part $\boldsymbol{A}^{\mathrm{s}}$ of the corresponding linear response matrix, while the odd torkance $\boldsymbol{t}^{\text {odd }}$ determines those of the antisymmetric part $\boldsymbol{A}^{\text {a }}$ :

$$
\begin{aligned}
& \left(\begin{array}{c}
j \\
\boldsymbol{T} / V
\end{array}\right)=\left[\boldsymbol{A}^{\mathrm{s}}(\hat{\boldsymbol{M}})+\boldsymbol{A}^{\mathrm{a}}(\hat{\boldsymbol{M}})\right]\left(\begin{array}{c}
\boldsymbol{E} \\
\hat{\boldsymbol{M}} \times \frac{d \hat{M}}{d t}
\end{array}\right) \\
& \boldsymbol{A}^{\mathrm{s}}(\hat{\boldsymbol{M}})=\left(\begin{array}{cc}
\boldsymbol{\sigma}^{\mathrm{even}}(\hat{\boldsymbol{M}}) & \left(\boldsymbol{t}^{\mathrm{even}}(\hat{\boldsymbol{M}})\right)^{\mathrm{T}} / V \\
\boldsymbol{t}^{\text {even }}(\hat{\boldsymbol{M}}) / V & -\boldsymbol{\Lambda}^{\mathrm{even}}(\hat{\boldsymbol{M}})
\end{array}\right) \\
& \boldsymbol{A}^{\mathrm{a}}(\hat{\boldsymbol{M}})=\left(\begin{array}{cc}
\boldsymbol{\sigma}^{\mathrm{odd}}(\hat{\boldsymbol{M}}) & -\left(\boldsymbol{t}^{\text {odd }}(\hat{\boldsymbol{M}})\right)^{\mathrm{T}} / V \\
\boldsymbol{t}^{\operatorname{odd}}(\hat{\boldsymbol{M}}) / V & -\boldsymbol{\Lambda}^{\mathrm{odd}}(\hat{\boldsymbol{M}})
\end{array}\right) .
\end{aligned}
$$

Here, $\boldsymbol{\sigma}$ is the tensor of electrical conductivity. The torque $\boldsymbol{T}$ in the first equation, i.e., $\boldsymbol{T}=\boldsymbol{t} \boldsymbol{E}-$ $V \boldsymbol{\Lambda}\left(\hat{M} \times \frac{d \hat{M}}{d t}\right)$, is the torque on the magnetization due to the response of the electrons to the two perturbations $\boldsymbol{E}$ and $\frac{d \hat{M}}{d t}$. According to Eq. (17) the sum of this torque and the torques due to magnetic anisotropy and external magnetic fields is zero. Due to the Onsager relation $\sigma_{\alpha \beta}(\hat{M})=\sigma_{\beta \alpha}(-\hat{M})$ the even part of the conductivity tensor is symmetric, i.e., $\sigma_{\alpha \beta}^{\text {even }}(\hat{\boldsymbol{M}})=\sigma_{\beta \alpha}^{\text {even }}(\hat{\boldsymbol{M}})$, while the odd part is antisymmetric, i.e., $\sigma_{\alpha \beta}^{\text {odd }}(\hat{M})=$ $-\sigma_{\beta \alpha}^{\text {odd }}(\hat{\boldsymbol{M}})$ [49]. Similarly, $\Lambda_{\alpha \beta}^{\text {even }}(\hat{\boldsymbol{M}})=\Lambda_{\beta \alpha}^{\text {even }}(\hat{\boldsymbol{M}})$ and $\Lambda_{\alpha \beta}^{\text {odd }}(\hat{M})=-\Lambda_{\beta \alpha}^{\text {odd }}(\hat{M})$. Consequently, $\boldsymbol{A}^{\mathrm{s}}(\hat{\boldsymbol{M}})$ is indeed symmetric and additionally even with respect to magnetization reversal. Likewise, $\boldsymbol{A}^{\mathrm{a}}(\hat{\boldsymbol{M}})$ is indeed antisymmetric and additionally odd with respect to magnetization reversal. Therefore, the linear response matrix $\boldsymbol{A}(\hat{\boldsymbol{M}})=\boldsymbol{A}^{\mathrm{s}}(\hat{\boldsymbol{M}})+\boldsymbol{A}^{\mathrm{a}}(\hat{\boldsymbol{M}})$ satisfies the symmetry

$$
(\boldsymbol{A}(\hat{\boldsymbol{M}}))^{\mathrm{T}}=\boldsymbol{A}(-\hat{\boldsymbol{M}}),
$$

which summarizes the Onsager relations of $\boldsymbol{\sigma}, \boldsymbol{\Lambda}$ and $\boldsymbol{t}$ in a compact form.

Eq. (16) and Eq. (22) are the central results of this subsection. They show that Gilbert damping $\boldsymbol{\alpha}$ (Eq. (20)), gyromagnetic ratio $\gamma$ (Eq. (19)) as well as ISOT (Eq. (59)) can be extracted coherently and consistently from timedependent perturbation theory, where the perturbation due to magnetization dynamics is given by Eq. (4).

From the point of view of adiabatic electron dynamics in a time-dependent Hamiltonian, Eq. (11), it is natural to consider the precession of the exchange field as perturbation. The electronic system responds to this perturbation by the ISOT current, Eq. (5). Additionally, it responds by the torque $\boldsymbol{T}=-V \boldsymbol{\Lambda}\left(\hat{\boldsymbol{M}} \times \frac{d \hat{M}}{d t}\right)$ described by Eq. (16). However, when the Onsager reciprocity principle is used to relate SOT and ISOT in a phenomenological approach typically a different point of view is taken: The effective magnetic field $\boldsymbol{H}^{\text {eff }}$ is considered as a thermodynamic force and the time-derivative of magnetization plays the role of the associated thermodynamic flux [19]. Instead of considering the response of $(\boldsymbol{j}, \boldsymbol{T} / V)^{\mathrm{T}}$ to the perturbation $\left(\boldsymbol{E}, \hat{\boldsymbol{M}} \times \frac{d \hat{\boldsymbol{M}}}{d t}\right)^{\mathrm{T}}$ as we do in Eq. (22) one considers then instead the response of the thermodynamic fluxes $\left(\frac{d \hat{\boldsymbol{M}}}{d t}, \boldsymbol{j}\right)^{\mathrm{T}}$ to the thermodynamic forces $\left(\boldsymbol{H}^{\mathrm{eff}}, \boldsymbol{E}\right)^{\mathrm{T}}$. Interestingly, $\frac{d \hat{M}}{d t}$ appears then as a response rather than as a perturbation. However, both formulations of the reciprocity between SOT and ISOT are equivalent.

\section{Many-electron response functions}

In the previous two subsections we discussed SOT and ISOT based on the effective single-particle Hamiltonian defined in Eq. (1), where the exchange field $\Omega^{\mathrm{xc}}(\boldsymbol{r})$ needs to be obtained self-consistently within LSDA. In this subsection we consider SOT and ISOT from the interacting many-electron point of view.

When a small static electric field $\boldsymbol{E}$ is applied to a magnet with broken inversion symmetry its magnetization will assume a new direction $\hat{M}+\delta \hat{M}$ due to the action of the SOT. We assume that $\boldsymbol{E}$ is sufficiently small to ensure that the magnetization is not switched and that $\hat{M}+\delta \hat{M}$ is time-independent. Within linear response the relation between $\delta \hat{M}$ and $\boldsymbol{E}$ is given by

$$
\delta \hat{\boldsymbol{M}}=\frac{1}{M V} \boldsymbol{\Xi}(\hat{\boldsymbol{M}}) \boldsymbol{E}
$$

with

$$
\Xi_{\alpha \beta}(\hat{\boldsymbol{M}})=\lim _{\omega \rightarrow 0} \frac{e}{i \omega \hbar} \mathcal{G}_{m_{\alpha}, v_{\beta}}^{\mathrm{R}}(\hbar \omega, \hat{\boldsymbol{M}}),
$$

where

$$
\mathcal{G}_{m_{\alpha}, v_{\beta}}^{\mathrm{R}}(\hbar \omega, \hat{\boldsymbol{M}})=-i \int_{0}^{\infty} d t e^{i \omega t}\left\langle\left[m_{\alpha}(t), v_{\beta}(0)\right]_{-}\right\rangle
$$

is the retarded spin-moment velocity correlation function. While the correlation functions defined in Eq. (6), Eq. (9) and Eq. (16) are evaluated based on the Kohn-Sham eigenfunctions of the effective single-particle Hamiltonian Eq. (7), Eq. (26) has to be evaluated based on the interacting many-electron wave functions of the 
system, i.e.,

$$
\begin{aligned}
\mathcal{G}_{m_{\alpha}, v_{\beta}}^{\mathrm{R}}(\hbar \omega, \hat{\boldsymbol{M}})= & \sum_{n} \hbar\left[\frac{\left\langle\Psi_{0}\left|m_{\alpha}\right| \Psi_{n}\right\rangle\left\langle\Psi_{n}\left|v_{\beta}\right| \Psi_{0}\right\rangle}{\mathcal{E}_{0}-\mathcal{E}_{n}+\hbar \omega+i \eta}\right. \\
& \left.-\frac{\left\langle\Psi_{0}\left|v_{\beta}\right| \Psi_{n}\right\rangle\left\langle\Psi_{n}\left|m_{\alpha}\right| \Psi_{0}\right\rangle}{\mathcal{E}_{n}-\mathcal{E}_{0}+\hbar \omega+i \eta}\right],
\end{aligned}
$$

where $\Psi_{0}$ is the ground state and $\Psi_{n}$ with $n>0$ are the excited states. The energies of the ground state and of the excited states are $\mathcal{E}_{0}$ and $\mathcal{E}_{n}$, respectively. We use the symbol $\mathcal{G}^{\mathrm{R}}$ to denote the retarded many-electron response functions while we use $G^{\mathrm{R}}$ to denote the retarded Kohn-Sham single-particle response functions.

We can quantify the SOT that gives rise to the rotation of magnetization $\delta \hat{M}$ in Eq. (24) in terms of the magnetic field $\boldsymbol{H}^{\text {SOT }}$ that would need to be applied perpendicular to $\hat{\boldsymbol{M}}$ to achieve the same tilt $\delta \hat{\boldsymbol{M}}$ without applied electric field $\boldsymbol{E}$. The relation between $\delta \hat{\boldsymbol{M}}$ and $\boldsymbol{H}^{\mathrm{SOT}}$ is described by the transverse magnetic susceptibility $\chi$ :

$$
M \delta \hat{\boldsymbol{M}}=\chi(\hat{\boldsymbol{M}}) \boldsymbol{H}^{\mathrm{SOT}}
$$

where

$$
\chi_{\alpha \beta}(\hat{\boldsymbol{M}})=-\frac{\mu_{0}}{V \hbar} \mathcal{G}_{m_{\alpha}, m_{\beta}}^{\mathrm{R}}(\hbar \omega=0, \hat{\boldsymbol{M}})
$$

The static transverse magnetic susceptibility $\chi(\hat{M})$ contains the information on the magnetic anisotropy [50]: When the magnetization is tilted away from the easy axis due to the applied transverse magnetic field $\boldsymbol{H}^{\mathrm{SOT}}$, the additional internal magnetic field

$$
\boldsymbol{H}^{\mathrm{MAE}}=-M[\chi(\hat{\boldsymbol{M}})]^{-1} \delta \hat{\boldsymbol{M}}
$$

due to magnetic anisotropy acts on the magnetization. The tilt $\delta \hat{\boldsymbol{M}}$ is such that $\boldsymbol{H}^{\mathrm{MAE}}+\boldsymbol{H}^{\mathrm{SOT}}=0$. Equating the right-hand sides of Eq. (24) and Eq. (28) we obtain an expression for the magnetic field $\boldsymbol{H}^{\mathrm{SOT}}$ :

$$
\boldsymbol{H}^{\mathrm{SOT}}=\frac{1}{V}[\chi(\hat{\boldsymbol{M}})]^{-1} \boldsymbol{\Xi}(\hat{\boldsymbol{M}}) \boldsymbol{E} .
$$

This magnetic field exerts the torque $\mu_{0} M V \hat{\boldsymbol{M}} \times \boldsymbol{H}^{\mathrm{SOT}}$ on the magnetization. Exactly the same torque acts on the magnetization when the electric field $\boldsymbol{E}$ is applied instead of the magnetic field $\boldsymbol{H}^{\mathrm{SOT}}$, i.e., the SOT is given by $\mu_{0} M V \hat{\boldsymbol{M}} \times \boldsymbol{H}^{\mathrm{SOT}}$. The corresponding torkance can be written as

$$
\tilde{\boldsymbol{t}}(\hat{\boldsymbol{M}})=\mu_{0} M \hat{M} \times[\chi(\hat{M})]^{-1} \Xi(\hat{M}) .
$$

The applicability of Eq. (8) is restricted to LSDA, because it is based on the torque operator $\mathcal{T}$ and hence on the exchange field $\Omega^{\mathrm{xc}}(\boldsymbol{r})$. In contrast, Eq. (32) provides a general formulation of the torkance.

In order to show that Eq. (32) reduces to Eq. (8) within LSDA, i.e., $\tilde{\boldsymbol{t}}(\hat{\boldsymbol{M}})=\boldsymbol{t}(\hat{\boldsymbol{M}})$, we need to express the many-electron response functions $\Xi(\hat{M})$ and $\chi(\hat{M})$ through the corresponding single-particle Kohn-Sham response functions

$$
\Xi_{\alpha \beta}^{\mathrm{KS}}(\hat{\boldsymbol{M}}, \boldsymbol{r})=\lim _{\omega \rightarrow 0} \frac{e}{i \omega \hbar} G_{m_{\alpha}(\boldsymbol{r}), v_{\beta}}^{\mathrm{R}}(\hbar \omega, \hat{\boldsymbol{M}})
$$

and

$$
\chi_{\alpha \beta}^{\mathrm{KS}}\left(\hat{\boldsymbol{M}}, \boldsymbol{r}, \boldsymbol{r}^{\prime}\right)=-\frac{\mu_{0}}{\hbar} G_{m_{\alpha}(\boldsymbol{r}), m_{\beta}\left(\boldsymbol{r}^{\prime}\right)}^{\mathrm{R}}(\hbar \omega=0, \hat{\boldsymbol{M}}),
$$

where $m_{\alpha}(\boldsymbol{r})$ is the operator of spin magnetic moment density at position $\boldsymbol{r}$, i.e., $\int d^{3} r m_{\alpha}(\boldsymbol{r})=m_{\alpha}=-\mu_{\mathrm{B}} \sigma_{\alpha}$. When an electric field $\boldsymbol{E}$ is applied to the system the transverse component of the change of magnetization at position $\boldsymbol{r}$, i.e., $m(\boldsymbol{r}) \delta \hat{\boldsymbol{M}}(\boldsymbol{r})$, is described by the integral equation

$$
\begin{aligned}
& m(\boldsymbol{r}) \delta \hat{\boldsymbol{M}}(\boldsymbol{r})=\boldsymbol{\Xi}^{\mathrm{KS}}(\hat{\boldsymbol{M}}, \boldsymbol{r}) \boldsymbol{E}+ \\
& \quad+\frac{1}{\mu_{0}} \int d^{3} r^{\prime} \boldsymbol{\chi}^{\mathrm{KS}}\left(\hat{\boldsymbol{M}}, \boldsymbol{r}, \boldsymbol{r}^{\prime}\right) \Omega^{\mathrm{xc}}\left(\boldsymbol{r}^{\prime}\right) \delta \hat{\boldsymbol{M}}\left(\boldsymbol{r}^{\prime}\right) .
\end{aligned}
$$

The second term on the right hand side takes into account that within LSDA the quasiparticles respond not only to the applied fields but also to the induced fields. In order to solve this integral equation approximatively, we assume that the change of magnetization direction is independent of position, i.e., $\delta \hat{M}(\boldsymbol{r})=\delta \hat{M}$. Multiplying both sides of Eq. (35) by $\Omega^{\mathrm{xc}}(\boldsymbol{r}) \hat{\boldsymbol{M}} \times$ from the left, and integrating over position $\boldsymbol{r}$ we obtain

$$
\begin{aligned}
& \bar{\Omega}^{\mathrm{xc}} M V(\hat{\boldsymbol{M}} \times \delta \hat{\boldsymbol{M}})=\boldsymbol{t}(\hat{\boldsymbol{M}}) \boldsymbol{E} \\
& -\frac{1}{\hbar} \sum_{\alpha \beta} \hat{\boldsymbol{e}}_{\alpha} G_{\mathcal{T}_{\alpha}}^{\mathrm{R}} \mathcal{T}_{\beta}(\hbar \omega=0, \hat{\boldsymbol{M}})[\hat{\boldsymbol{M}} \times \delta \hat{\boldsymbol{M}}]_{\beta} .
\end{aligned}
$$

The average exchange field on the left-hand side is defined as

$$
\bar{\Omega}^{\mathrm{xc}}=\frac{\int d^{3} r \Omega^{\mathrm{xc}}(\boldsymbol{r}) m(\boldsymbol{r})}{\int d^{3} r^{\prime} m\left(\boldsymbol{r}^{\prime}\right)}=\frac{\int d^{3} r \Omega^{\mathrm{xc}}(\boldsymbol{r}) m(\boldsymbol{r})}{M V} .
$$

To obtain the first term on the right-hand side of Eq. (36) we made use of

$$
\hat{\boldsymbol{M}} \times \int d^{3} r \boldsymbol{\Xi}^{\mathrm{KS}}(\hat{\boldsymbol{M}}, \boldsymbol{r}) \Omega^{\mathrm{xc}}(\boldsymbol{r})=\boldsymbol{t}
$$

which follows from comparison of Eq. (8) and Eq. (33). Solving Eq. (36) for $\delta \hat{M}$ and comparing to Eq. (24) yields the following expression for $\boldsymbol{\Xi}(\hat{M})$ :

$$
\boldsymbol{\Xi}(\hat{\boldsymbol{M}})=-\hat{\boldsymbol{M}} \times\left[\bar{\Omega}^{\mathrm{xc}}+\frac{G_{\mathcal{T}}^{\mathrm{R}}(\hbar \omega=0, \hat{\boldsymbol{M}})}{M V \hbar}\right]^{-1} \boldsymbol{t}(\hat{\boldsymbol{M}})
$$

In order to obtain an expression for $\chi(\hat{\boldsymbol{M}})$ in Eq. (28) we need to replace $\boldsymbol{\Xi}^{\mathrm{KS}}(\hat{\boldsymbol{M}}, \boldsymbol{r}) \boldsymbol{E}$ in Eq. (35) by $\int d^{3} r^{\prime} \chi^{\mathrm{KS}}\left(\hat{\boldsymbol{M}}, \boldsymbol{r}, \boldsymbol{r}^{\prime}\right) \boldsymbol{H}^{\mathrm{SOT}}$, which yields the equation

$$
\begin{aligned}
& m(\boldsymbol{r}) \delta \hat{\boldsymbol{M}}(\boldsymbol{r})=\int d^{3} r^{\prime} \boldsymbol{\chi}^{\mathrm{KS}}\left(\hat{\boldsymbol{M}}, \boldsymbol{r}, \boldsymbol{r}^{\prime}\right) \Omega^{\mathrm{xc}}\left(\boldsymbol{r}^{\prime}\right) \frac{\boldsymbol{H}^{\mathrm{SOT}}}{\bar{\Omega}^{\mathrm{xc}}}+ \\
& +\frac{1}{\mu_{0}} \int d^{3} r^{\prime} \chi^{\mathrm{KS}}\left(\hat{\boldsymbol{M}}, \boldsymbol{r}, \boldsymbol{r}^{\prime}\right) \Omega^{\mathrm{xc}}\left(\boldsymbol{r}^{\prime}\right) \delta \hat{\boldsymbol{M}}\left(\boldsymbol{r}^{\prime}\right)
\end{aligned}
$$


where we replaced the magnetic field $\boldsymbol{H}^{\mathrm{SOT}}$ by $\boldsymbol{H}^{\mathrm{SOT}} \Omega^{\mathrm{xc}}\left(\boldsymbol{r}^{\prime}\right) / \bar{\Omega}^{\mathrm{xc}}$, because both magnetic fields produce the same torque on the magnetization $[50]$ :

$$
\frac{\mu_{0} \hat{\boldsymbol{M}} \times \boldsymbol{H}^{\mathrm{SOT}}}{\bar{\Omega}^{\mathrm{xc}}} \int d^{3} r m(\boldsymbol{r}) \Omega^{\mathrm{xc}}(\boldsymbol{r})=\mu_{0} V \boldsymbol{M} \times \boldsymbol{H}^{\mathrm{SOT}} .
$$

Multiplying both sides of Eq. (40) by $\Omega^{\mathrm{xc}}(\boldsymbol{r}) \hat{\boldsymbol{M}} \times$ from the left, and integrating over position $\boldsymbol{r}$, we obtain

$$
\begin{aligned}
& \bar{\Omega}^{\mathrm{xc}} M V(\hat{\boldsymbol{M}} \times \delta \hat{\boldsymbol{M}})= \\
& -\frac{\mu_{0}}{\hbar \bar{\Omega}^{\mathrm{xc}}} \sum_{\alpha \beta} \hat{\boldsymbol{e}}_{\alpha} G_{\mathcal{T}_{\alpha}}^{\mathrm{R}} \mathcal{T}_{\beta}(\hbar \omega=0, \hat{\boldsymbol{M}})\left[\hat{\boldsymbol{M}} \times \boldsymbol{H}^{\mathrm{SOT}}\right]_{\beta} \\
& -\frac{1}{\hbar} \sum_{\alpha \beta} \hat{\boldsymbol{e}}_{\alpha} G_{\mathcal{T}_{\alpha}}^{\mathrm{R}} \mathcal{T}_{\beta}(\hbar \omega=0, \hat{\boldsymbol{M}})[\hat{\boldsymbol{M}} \times \delta \hat{\boldsymbol{M}}]_{\beta} .
\end{aligned}
$$

Comparing Eq. (42) and Eq. (36) leads to

$$
\boldsymbol{t}(\hat{\boldsymbol{M}}) \boldsymbol{E}=-\frac{\mu_{0}}{\hbar \bar{\Omega}^{\mathrm{xc}}} G_{\mathcal{T}}^{\mathrm{R}}(\hbar \omega=0, \hat{\boldsymbol{M}})\left[\hat{\boldsymbol{M}} \times \boldsymbol{H}^{\mathrm{SOT}}\right] .
$$

In the absence of SOI, $G_{\mathcal{T} \mathcal{T}}^{\mathrm{R}}(\hbar \omega=0, \hat{\boldsymbol{M}})$ is given by (see Appendix A

$$
G_{\mathcal{T} \mathcal{T}}^{\mathrm{R}}(\hbar \omega=0, \hat{\boldsymbol{M}})=-\hbar M V \bar{\Omega}^{\mathrm{xc}}\left[1-\hat{\boldsymbol{M}}^{\mathrm{T}} \hat{\boldsymbol{M}}\right] .
$$

We assume that the magnetic anisotropy is small compared to the exchange splitting. In this case we can approximate $G_{\mathcal{T}}^{\mathrm{R}}(\hbar \omega=0, \hat{\boldsymbol{M}})$ in Eq. (43) by Eq. (44) and obtain

$$
\boldsymbol{T}^{\mathrm{SOT}}=\mu_{0} M V\left[\hat{\boldsymbol{M}} \times \boldsymbol{H}^{\mathrm{SOT}}\right]=\boldsymbol{t}(\hat{\boldsymbol{M}}) \boldsymbol{E} .
$$

Eq. (45) shows that the description of the SOT through Eq. (32) in terms of many-electron response functions Eq. (25) and Eq. (29) recovers the single-particle expression Eq. (8).

Solving Eq. (42) for $\delta \hat{M}$ and comparing to Eq. (28) yields

$$
\begin{aligned}
& \chi(\hat{\boldsymbol{M}})=\frac{\mu_{0}}{\hbar V \bar{\Omega}^{\mathrm{xc}}} \hat{\boldsymbol{M}} \times\left[\bar{\Omega}^{\mathrm{xc}}+\right. \\
& \left.+\frac{G_{\mathcal{T} \mathcal{T}}^{\mathrm{R}}(\hbar \omega=0, \hat{\boldsymbol{M}})}{M V \hbar}\right]^{-1} G_{\mathcal{\mathcal { T }}}^{\mathrm{R}}(\hbar \omega=0, \hat{\boldsymbol{M}}) \hat{\boldsymbol{M}} \times,
\end{aligned}
$$

where $\hat{M} \times$ is a shorthand for the matrix

$$
\frac{1}{M}\left(\begin{array}{ccc}
0 & -M_{3} & M_{2} \\
M_{3} & 0 & -M_{1} \\
-M_{2} & M_{1} & 0
\end{array}\right)=\hat{M} \times
$$

Assuming that the anisotropy energy is much smaller than the exchange splitting, we can approximate the rightmost $G_{\mathcal{T}}^{\mathrm{R}}$ in Eq. (46) by Eq. (44) and obtain

$$
\chi(\hat{\boldsymbol{M}})=-\mu_{0} M \hat{\boldsymbol{M}} \times\left[\bar{\Omega}^{\mathrm{xc}}+\frac{G_{\mathcal{T} \mathcal{T}}^{\mathrm{R}}(\hbar \omega=0, \hat{\boldsymbol{M}})}{M V \hbar}\right]^{-1} \hat{\boldsymbol{M}} \times
$$

The difference between the right-hand side and the lefthand side of Eq. (44) describes the magnetic anisotropy (see Appendix $\mathrm{A}$ ). Therefore, the remaining $G_{\mathcal{T}}^{\mathrm{R}} \mathcal{i n}$ Eq. (48) cannot be approximated by Eq. (44). Inserting Eq. (48) and Eq. (39) into Eq. (32) leads to the identity $\tilde{\boldsymbol{t}}(\hat{\boldsymbol{M}})=\boldsymbol{t}(\hat{\boldsymbol{M}})$, showing again the equivalence between the single-particle and the many-electron expressions, Eq. (8) and Eq. (32), respectively.

Using Eq. (48) we can rewrite Eq. (39) as

$$
\boldsymbol{\Xi}(\hat{\boldsymbol{M}})=-\frac{1}{\mu_{0} M} \chi(\hat{\boldsymbol{M}}) \hat{\boldsymbol{M}} \times \boldsymbol{t}(\hat{\boldsymbol{M}}) .
$$

In this expression, $\hat{\boldsymbol{M}} \times \boldsymbol{t}(\hat{\boldsymbol{M}})$ on the right-hand side can be interpreted in terms of a current-induced effective magnetic field $\boldsymbol{H}^{\mathrm{SOT}}=-[\hat{\boldsymbol{M}} \times \boldsymbol{t}(\hat{\boldsymbol{M}}) \boldsymbol{E}] /\left(M V \mu_{0}\right)$. The transverse magnetic susceptibility $\chi(\hat{M})$ describes the response of the magnetization to $\boldsymbol{H}^{\mathrm{SOT}}$.

Next, we consider the generation of a current density $j$ due to a time-dependent applied magnetic field $\boldsymbol{H}^{\operatorname{ext}}(\omega, t)=\boldsymbol{H}^{\mathrm{ext}}(\omega) e^{-i \omega t}$. Denoting the corresponding linear response tensor by $\boldsymbol{\Phi}(\hat{\boldsymbol{M}}, \omega)$ we can write

$$
\begin{aligned}
\boldsymbol{j} & =\boldsymbol{\Phi}(\hat{\boldsymbol{M}}, \omega) \boldsymbol{H}^{\mathrm{ext}}(\omega) e^{-i \omega t} \\
& \left.\simeq \frac{d \boldsymbol{\Phi}(\hat{\boldsymbol{M}}, \omega)}{d \omega}\right|_{\omega=0} \omega \boldsymbol{H}^{\mathrm{ext}}(\omega) e^{-i \omega t} \\
& =i \boldsymbol{\Phi}^{\prime}(\hat{\boldsymbol{M}}) \frac{d \boldsymbol{H}^{\operatorname{ext}}(\omega, t)}{d t}
\end{aligned}
$$

where $\boldsymbol{\Phi}^{\prime}(\hat{\boldsymbol{M}})$ denotes the frequency derivative, i.e., $\boldsymbol{\Phi}^{\prime}(\hat{\boldsymbol{M}})=\left.\frac{d \boldsymbol{\Phi}(\hat{M}, \omega)}{d \omega}\right|_{\omega=0}$. We used that $\boldsymbol{\Phi}(\hat{\boldsymbol{M}}, \omega=0)$ does not generate an ISOT current and we expanded $\boldsymbol{\Phi}(\hat{\boldsymbol{M}}, \omega)$ up to first order in frequency. Assuming that the field $\boldsymbol{H}^{\operatorname{ext}}(\omega, t)$ is transverse to magnetization, we can use the transverse magnetic susceptibility $\chi(\hat{\boldsymbol{M}})$, Eq. (28), to express it in terms of the corresponding tilt of the magnetization direction. This allows us to relate $j$ to the time-derivative of the magnetization direction:

$$
\begin{aligned}
j & =i M \boldsymbol{\Phi}^{\prime}(\hat{\boldsymbol{M}})[\chi(\hat{\boldsymbol{M}})]^{-1} \frac{d \hat{\boldsymbol{M}}}{d t} \\
& =-i M \boldsymbol{\Phi}^{\prime}(\hat{\boldsymbol{M}})[\chi(\hat{\boldsymbol{M}})]^{-1} \hat{\boldsymbol{M}} \times\left[\hat{\boldsymbol{M}} \times \frac{d \hat{\boldsymbol{M}}}{d t}\right] .
\end{aligned}
$$

We can use the retarded velocity spin-moment correlation function

$$
\mathcal{G}_{v_{\alpha}, m_{\beta}}^{\mathrm{R}}(\hbar \omega, \hat{M})=-i \int_{0}^{\infty} d t e^{i \omega t}\left\langle\left[v_{\alpha}(t), m_{\beta}(0)\right]_{-}\right\rangle
$$

to express $\boldsymbol{\Phi}^{\prime}(\hat{\boldsymbol{M}})$ as follows:

$$
\Phi_{\alpha \beta}^{\prime}(\hat{\boldsymbol{M}})=\lim _{\omega \rightarrow 0} \frac{d}{d \omega} \frac{e \mu_{0}}{\hbar V} \mathcal{G}_{v_{\alpha}, m_{\beta}}^{\mathrm{R}}(\hbar \omega, \hat{\boldsymbol{M}})
$$


The spectral densities of the Green functions defined in Eq. (26) and in Eq. (52) are given by

$$
\begin{aligned}
& S_{m_{\alpha}, v_{\beta}}\left(t, t^{\prime}, \hat{\boldsymbol{M}}\right)=\frac{1}{2 \pi}\left\langle\left[m_{\alpha}(t), v_{\beta}\left(t^{\prime}\right)\right]_{-}\right\rangle, \\
& S_{v_{\alpha}, m_{\beta}}\left(t, t^{\prime}, \hat{\boldsymbol{M}}\right)=\frac{1}{2 \pi}\left\langle\left[v_{\alpha}(t), m_{\beta}\left(t^{\prime}\right)\right]_{-}\right\rangle
\end{aligned}
$$

and their Fourier transforms satisfy the relations

$$
\begin{aligned}
S_{m_{\alpha}, v_{\beta}}(\hbar \omega, \hat{\boldsymbol{M}}) & =\left[S_{v_{\beta}, m_{\alpha}}(\hbar \omega, \hat{\boldsymbol{M}})\right]^{*}, \\
\operatorname{Re}\left[S_{v_{\beta}, m_{\alpha}}(\hbar \omega,-\hat{\boldsymbol{M}})\right] & =\operatorname{Re}\left[S_{v_{\beta}, m_{\alpha}}(\hbar \omega, \hat{\boldsymbol{M}})\right], \\
\operatorname{Im}\left[S_{v_{\beta}, m_{\alpha}}(\hbar \omega,-\hat{\boldsymbol{M}})\right] & =-\operatorname{Im}\left[S_{v_{\beta}, m_{\alpha}}(\hbar \omega, \hat{\boldsymbol{M}})\right],
\end{aligned}
$$

from which follows

$$
S_{m_{\alpha}, v_{\beta}}(\hbar \omega, \hat{\boldsymbol{M}})=S_{v_{\beta}, m_{\alpha}}(\hbar \omega,-\hat{\boldsymbol{M}})
$$

and thus

$$
\mathcal{G}_{m_{\alpha}, v_{\beta}}^{\mathrm{R}}(\hbar \omega, \hat{\boldsymbol{M}})=\mathcal{G}_{v_{\beta}, m_{\alpha}}^{\mathrm{R}}(\hbar \omega,-\hat{\boldsymbol{M}})
$$

and

$$
\boldsymbol{\Phi}^{\prime}(\hat{\boldsymbol{M}})=\lim _{\omega \rightarrow 0} \frac{d}{d \omega} \frac{e \mu_{0}}{\hbar V}\left[\mathcal{G}_{\boldsymbol{m}, \boldsymbol{v}}^{\mathrm{R}}(\hbar \omega,-\hat{\boldsymbol{M}})\right]^{\mathrm{T}} .
$$

Using Eq. (32), $[\hat{\boldsymbol{M}} \times]^{\mathrm{T}}=-\hat{\boldsymbol{M}} \times($ see Eq. (47)) $)$ and the Onsager relation $\chi(\hat{\boldsymbol{M}})=[\chi(-\hat{\boldsymbol{M}})]^{\mathrm{T}}$ we can relate $\boldsymbol{\Phi}^{\prime}(\hat{\boldsymbol{M}})$ and the torkance $\tilde{\boldsymbol{t}}(\hat{\boldsymbol{M}})$ as follows:

$$
[\tilde{\boldsymbol{t}}(-\hat{\boldsymbol{M}})]^{\mathrm{T}}=-i V M \boldsymbol{\Phi}^{\prime}(\hat{\boldsymbol{M}})[\boldsymbol{\chi}(\hat{\boldsymbol{M}})]^{-1} \hat{\boldsymbol{M}} \times .
$$

This allows us to rewrite Eq. (51) as

$$
\boldsymbol{j}=\frac{1}{V}[\tilde{\boldsymbol{t}}(-\hat{\boldsymbol{M}})]^{\mathrm{T}} \hat{\boldsymbol{M}} \times \frac{d \boldsymbol{M}}{d t}
$$

in agreement with Eq. (14) derived earlier in the singleparticle formalism.

The central result of this subsection is Eq. (32), which provides a general definition of the torkance that is not limited to the framework of Kohn-Sham theory. The reciprocity between direct and inverse SOT as discussed in the previous subsection based on Kohn-Sham theory remains valid within the many-electron response function formalism used in this subsection.

\section{SOT AND ISOT IN BILAYER SYSTEMS}

In the following we discuss SOT and ISOT in magnetic bilayer systems composed of a ferromagnetic layer (FM) deposited on a normal metal (NM). When the electric field $\boldsymbol{E}=\mathrm{E}_{x} \hat{\boldsymbol{e}}_{x}$ is applied in-plane along $x$ direction, the torques satisfy

$$
\begin{gathered}
\boldsymbol{T}^{\mathrm{even}}(\hat{\boldsymbol{M}})=\mathrm{E}_{x} \hat{\boldsymbol{M}} \times\left(\hat{\boldsymbol{e}}_{y} \times \hat{\boldsymbol{M}}\right)\left[A_{0}+A_{2}\left(\hat{\boldsymbol{e}}_{z} \times \hat{\boldsymbol{M}}\right)^{2}+\ldots\right]+ \\
+\mathrm{E}_{x}\left(\hat{\boldsymbol{M}} \times \hat{\boldsymbol{e}}_{z}\right)\left(\hat{\boldsymbol{M}} \cdot \hat{\boldsymbol{e}}_{x}\right)\left[B_{2}+B_{4}\left(\hat{\boldsymbol{e}}_{z} \times \hat{\boldsymbol{M}}\right)^{2}+\ldots\right]
\end{gathered}
$$

and

$$
\begin{aligned}
& \boldsymbol{T}^{\mathrm{odd}}(\hat{\boldsymbol{M}})=\mathrm{E}_{x}\left(\hat{\boldsymbol{e}}_{y} \times \hat{\boldsymbol{M}}\right)\left[C_{0}+C_{2}\left(\hat{\boldsymbol{e}}_{z} \times \hat{\boldsymbol{M}}\right)^{2}+\ldots\right]+ \\
& \quad+\mathrm{E}_{x} \hat{\boldsymbol{M}} \times\left(\hat{\boldsymbol{M}} \times \hat{\boldsymbol{e}}_{z}\right)\left(\hat{\boldsymbol{M}} \cdot \hat{\boldsymbol{e}}_{x}\right)\left[D_{2}+D_{4}\left(\boldsymbol{e}_{z} \times \hat{\boldsymbol{M}}\right)^{2}+\ldots\right]
\end{aligned}
$$

in bilayer systems composed of polycrystalline, disordered or amorphous layers with continuous rotational symmetry around the $z$ axis [24].

To describe the ISOT in bilayer systems we consider instead of the current density $j_{\alpha}$ the current per length $J_{\alpha}$, which is obtained by replacing the current density operator $-e v_{\alpha} / V$ by $-e v_{\alpha} / A$, where $A$ is the cross sectional area of the unit cell of the bilayer normal to the stacking direction:

$$
J_{\alpha}(t)=\frac{1}{A} \sum_{\beta} t_{\beta \alpha}(-\hat{\boldsymbol{M}}(t))\left(\hat{\boldsymbol{M}}(t) \times \frac{d \hat{\boldsymbol{M}}(t)}{d t}\right)_{\beta} .
$$

Since the atom-resolved current is expected to vary significantly between atomic layers in bilayer systems, $J_{\alpha}$ is a suitable definition of current density in such systems. In terms of $\boldsymbol{J}$, the electric current flowing in $x$ direction is given by $I_{x}=J_{x} L_{y}$, where $L_{y}$ is the length of the system in $y$ direction, and similarly $I_{y}=J_{y} L_{x}$ is the electric current in $y$ direction. Separating $J_{\alpha}$ into the components due to $\boldsymbol{t}^{\text {even }}(\hat{\boldsymbol{M}})$ and $\boldsymbol{t}^{\text {odd }}(\hat{\boldsymbol{M}})$ yields

$$
\begin{aligned}
J_{\alpha}^{\text {even }}(t) & =\frac{1}{A} \sum_{\beta} t_{\beta \alpha}^{\text {even }}(\hat{\boldsymbol{M}}(t))\left(\hat{\boldsymbol{M}}(t) \times \frac{d \hat{\boldsymbol{M}}(t)}{d t}\right)_{\beta}, \\
J_{\alpha}^{\text {odd }}(t) & =-\frac{1}{A} \sum_{\beta} t_{\beta \alpha}^{\text {odd }}(\hat{\boldsymbol{M}}(t))\left(\hat{\boldsymbol{M}}(t) \times \frac{d \hat{\boldsymbol{M}}(t)}{d t}\right)_{\beta} .
\end{aligned}
$$

In the following we discuss the magnetizationdynamics induced current density $J_{x}$ in $x$ direction. Using Eq. (61) and Eq. (62) in Eq. (64) we obtain

$$
\begin{aligned}
J_{x}^{\text {even }}( & t)=\frac{A_{0}}{A}\left[\hat{\boldsymbol{M}} \times\left(\hat{\boldsymbol{e}}_{y} \times \hat{\boldsymbol{M}}\right)\right] \cdot\left[\hat{\boldsymbol{M}} \times \frac{d \hat{\boldsymbol{M}}}{d t}\right]+ \\
+ & \frac{A_{2}}{A}\left[\hat{\boldsymbol{M}} \times\left(\hat{\boldsymbol{e}}_{y} \times \hat{\boldsymbol{M}}\right)\right] \cdot\left[\hat{\boldsymbol{M}} \times \frac{d \hat{\boldsymbol{M}}}{d t}\right]\left(\hat{\boldsymbol{e}}_{z} \times \hat{\boldsymbol{M}}\right)^{2}+ \\
+ & \frac{B_{2}}{A}\left(\hat{\boldsymbol{M}} \times \hat{\boldsymbol{e}}_{z}\right) \cdot\left[\hat{\boldsymbol{M}} \times \frac{d \hat{\boldsymbol{M}}}{d t}\right]\left(\hat{\boldsymbol{M}} \cdot \hat{\boldsymbol{e}}_{x}\right)+ \\
+ & \frac{B_{4}}{A}\left(\hat{\boldsymbol{M}} \times \hat{\boldsymbol{e}}_{z}\right) \cdot\left[\hat{\boldsymbol{M}} \times \frac{d \hat{\boldsymbol{M}}}{d t}\right]\left(\hat{\boldsymbol{M}} \cdot \hat{\boldsymbol{e}}_{x}\right)\left(\hat{\boldsymbol{e}}_{z} \times \hat{\boldsymbol{M}}\right)^{2}+ \\
+ & \cdots
\end{aligned}
$$


and

$$
\begin{aligned}
J_{x}^{\text {odd }}(t) & =-\frac{C_{0}}{A}\left(\hat{\boldsymbol{e}}_{y} \times \hat{\boldsymbol{M}}\right) \cdot\left[\hat{\boldsymbol{M}} \times \frac{d \hat{\boldsymbol{M}}}{d t}\right] \\
- & \frac{C_{2}}{A}\left(\hat{\boldsymbol{e}}_{y} \times \hat{\boldsymbol{M}}\right) \cdot\left[\hat{\boldsymbol{M}} \times \frac{d \hat{\boldsymbol{M}}}{d t}\right]\left(\hat{\boldsymbol{e}}_{z} \times \hat{\boldsymbol{M}}\right)^{2} \\
- & \frac{D_{2}}{A}\left[\hat{\boldsymbol{M}} \times\left(\hat{\boldsymbol{M}} \times \hat{\boldsymbol{e}}_{z}\right)\right] \cdot\left[\hat{\boldsymbol{M}} \times \frac{d \hat{\boldsymbol{M}}}{d t}\right]\left(\hat{\boldsymbol{M}} \cdot \hat{\boldsymbol{e}}_{x}\right) \\
& -\frac{D_{4}}{A}\left[\hat{\boldsymbol{M}} \times\left(\hat{\boldsymbol{M}} \times \hat{\boldsymbol{e}}_{z}\right)\right] \cdot\left[\hat{\boldsymbol{M}} \times \frac{d \hat{\boldsymbol{M}}}{d t}\right]\left(\hat{\boldsymbol{M}} \cdot \hat{\boldsymbol{e}}_{x}\right)\left(\hat{\boldsymbol{e}}_{z} \times \hat{\boldsymbol{M}}\right)^{2} \\
& -\cdots
\end{aligned}
$$

\section{A. Current densities induced by FMR through the inverse SOT}

First, we consider the case of FMR-driven magnetization precession around the $z$ axis in a circular orbit, i.e.,

$$
\hat{\boldsymbol{M}}(t)=[\sin (\theta) \cos (\omega t), \sin (\theta) \sin (\omega t), \cos (\theta)]^{\mathrm{T}},
$$

where $\theta$ is the cone angle. Inserting Eq. (67) into Eqs. (65) and (66) we obtain

$$
\begin{aligned}
J_{x}^{\text {even }}(t) & =-\frac{\omega}{A} \sin (\theta) \cos (\theta) \sin (\omega t)\left[A_{0}+A_{2} \sin ^{2}(\theta)+\cdots\right], \\
J_{x}^{\text {odd }}(t) & =\frac{\omega}{A} \sin (\theta) \cos (\omega t)\left[C_{0}+C_{2} \sin ^{2}(\theta)+\cdots\right]+ \\
+ & \frac{\omega}{A} \sin (\theta) \cos (\omega t)\left[D_{2} \sin ^{2}(\theta)+D_{4} \sin ^{4}(\theta)+\cdots\right] .
\end{aligned}
$$

For small cone angles $\theta$ the $\sin ^{2}(\theta)$ factors suppress the contributions from $A_{2}, C_{2}, D_{2}$ and further higher-order terms. In the small cone limit the ISOT for magnetization precession around the $z$ axis can thus be expressed in terms of the torkance for magnetization along $z$, if $A_{0}=t_{y x}^{\text {even }}\left(\hat{\boldsymbol{M}}=\hat{\boldsymbol{e}}_{z}\right)$ and $C_{0}=t_{x x}^{\text {odd }}\left(\hat{\boldsymbol{M}}=\hat{\boldsymbol{e}}_{z}\right)$ are used. Experiments [24, 25] and ab initio calculations [31] have found that $A_{0}$ and $C_{0}$ can be of the same order of magnitude in $\mathrm{AlO}_{x} / \mathrm{Co} / \mathrm{Pt}$ and $\mathrm{MgO} / \mathrm{CoFeB} / \mathrm{Ta}$. The two contributions $J_{x}^{\text {even }}(t)$ and $J_{x}^{\text {odd }}(t)$ are therefore expected to exhibit similar amplitudes. Since $J_{x}^{\text {even }}(t) \propto \sin (\omega t)$ while $J_{x}^{\text {odd }}(t) \propto \cos (\omega t)$ the even and odd part are phaseshifted with respect to each other.

Next, we consider FMR-driven magnetization precession around the $y$ axis. In this case the magnetization follows an elliptical trajectory in thin bilayer films due to the demagnetizing field [51],

$$
\hat{\boldsymbol{M}}(t)=\frac{1}{\eta(t)}[\sin (\theta) \sin (\omega t) \epsilon, \cos (\theta), \sin (\theta) \cos (\omega t)]^{\mathrm{T}},
$$

where $\epsilon$ is the ratio of the major axis to the minor axis of the ellipse and normalization of $\hat{\boldsymbol{M}}(t)$ is assured by $\eta(t)=\sqrt{1+\left[\epsilon^{2}-1\right] \sin ^{2}(\omega t) \sin ^{2}(\theta)}$. The resulting induced current density is given by

$$
\begin{aligned}
J_{x}^{\text {even }}(t) & =\frac{\omega \epsilon \sin ^{2} \theta}{A \eta^{2}(t)}\left[A_{0}+A_{2} \frac{\eta^{2}(t)-\cos ^{2}(\omega t) \sin ^{2} \theta}{\eta^{2}(t)}+\cdots\right] \\
- & \frac{\omega \epsilon \sin ^{2} \theta \sin ^{2}(\omega t)}{A \eta^{4}(t)}\left[1+\sin ^{2} \theta\left(\epsilon^{2}-1\right)\right]\left[B_{2}+\right. \\
+ & \left.B_{4} \frac{\eta^{2}(t)-\cos ^{2}(\omega t) \sin ^{2} \theta}{\eta^{2}(t)}+\cdots\right], \\
J_{x}^{\text {odd }}(t) & =\frac{\omega\left(1-\epsilon^{2}\right)}{2 A \eta^{3}(t)} \sin ^{2} \theta \cos \theta \sin (2 \omega t)\left[C_{0}+\right. \\
& \left.+C_{2} \frac{\eta^{2}(t)-\cos ^{2}(\omega t) \sin ^{2} \theta}{\eta^{2}(t)}+\cdots\right] \\
& -\frac{\omega \epsilon^{2}}{2 A \eta^{3}(t)} \sin (2 \omega t) \sin ^{2} \theta \cos \theta\left[D_{2}+\right. \\
& \left.+D_{4} \frac{\eta^{2}(t)-\cos ^{2}(\omega t) \sin ^{2} \theta}{\eta^{2}(t)}+\cdots\right] .
\end{aligned}
$$

For small angles $\theta$ the terms proportional to $\sin ^{2} \theta$ dominate, while terms proportional to $\sin ^{4} \theta$ and higher are suppressed. Thus, we can approximate in the small-cone limit

$$
\begin{aligned}
J_{x}^{\text {even }}(t) & =\frac{\omega \epsilon}{A} \sin ^{2} \theta\left[A_{0}+A_{2}+A_{4}+\cdots\right] \\
& -\frac{\omega \epsilon}{2 A} \sin ^{2} \theta[1-\cos (2 \omega t)]\left[B_{2}+B_{4}+\cdots\right], \\
J_{x}^{\text {odd }}(t) & =\frac{\omega}{2 A} \sin ^{2} \theta \sin (2 \omega t)\left(1-\epsilon^{2}\right)\left[C_{0}+C_{2}+\cdots\right] \\
& -\frac{\omega}{2 A} \sin ^{2} \theta \sin (2 \omega t) \epsilon^{2}\left[D_{2}+D_{4}+\cdots\right] .
\end{aligned}
$$

$J_{x}^{\text {even }}$ is the sum of a dc component and an ac component with frequency $2 \omega$, while $J_{x}^{\text {odd }}$ consists of only an ac part with frequency $2 \omega$. The ac components of the even and odd part are phase shifted. Compared to the induced current for precession around the $z$ axis, Eq. (68), the amplitude is expected to be typically reduced by roughly a factor of $\sin \theta$ when the magnetization precesses around the $y$ axis. The dc component of the voltage $-R_{x x} J_{x}^{\text {even }} L_{y}$, where $R_{x x}$ is the resistance, has been measured for several bilayer systems and is usually interpreted as the voltage arising from the conversion of pumped dc spin current via the ISHE 34 36].

We turn now to the FMR-driven magnetization precession around the $x$ axis. Again, the magnetization follows an elliptical trajectory,

$$
\hat{M}(t)=\frac{1}{\tilde{\eta}(t)}[\cos (\theta), \sin (\theta) \cos (\omega t) \epsilon, \sin (\theta) \sin (\omega t)]^{\mathrm{T}},
$$

with $\epsilon$ the ratio of major axis to minor axis of the ellipse and $\tilde{\eta}(t)=\sqrt{1+\left[\epsilon^{2}-1\right] \cos ^{2}(\omega t) \sin ^{2}(\theta)}$. In this case the current density induced by the precessing magnetization 
is given by

$$
\begin{aligned}
& J_{x}^{\text {even }}(t)=-\frac{\omega}{2 A \tilde{\eta}^{2}(t)} \sin (2 \theta) \cos (\omega t)\left\{A_{0}+\right. \\
& \left.+\frac{A_{2}}{\tilde{\eta}^{2}(t)}\left[\cos ^{2} \theta+\epsilon^{2} \sin ^{2} \theta \cos ^{2}(\omega t)\right]+\cdots\right\}+ \\
& +\frac{\omega}{2 A \tilde{\eta}^{4}(t)} \sin (2 \theta) \cos (\omega t)\left[1+\sin ^{2} \theta\left(\epsilon^{2}-1\right)\right] \times \\
& \quad \times\left\{B_{2}+\frac{B_{4}}{\tilde{\eta}^{2}(t)}\left[\cos ^{2} \theta+\epsilon^{2} \sin ^{2} \theta \cos ^{2}(\omega t)\right]+\cdots\right\}, \\
& J_{x}^{\text {odd }}(t)=-\frac{\omega \epsilon}{A \tilde{\eta}^{3}(t)} \sin \theta \sin (\omega t)\left\{C_{0}+\right. \\
& \left.+\frac{C_{2}}{\tilde{\eta}^{2}(t)}\left[\cos ^{2} \theta+\epsilon^{2} \sin ^{2} \theta \cos ^{2}(\omega t)\right]+\cdots\right\} \\
& -\frac{\omega \epsilon}{A \tilde{\eta}^{3}(t)} \sin \theta \cos ^{2} \theta \sin (\omega t)\left\{D_{2}+\right. \\
& \left.+\frac{D_{4}}{\tilde{\eta}^{2}(t)}\left[\cos ^{2} \theta+\epsilon^{2} \sin ^{2} \theta \cos ^{2}(\omega t)\right]+\cdots\right\} .
\end{aligned}
$$

In the small-cone limit we obtain

$$
\begin{aligned}
J_{x}^{\text {even }}(t) & =\frac{\omega}{2 A} \sin (2 \theta) \cos (\omega t)\left[B_{2}+B_{4}+\cdots-A_{0}-A_{2}-\cdots\right]= \\
& =-t_{y x}^{\text {even }}\left(\hat{\boldsymbol{M}}=\hat{\boldsymbol{e}}_{x}\right) \frac{\omega}{2 A} \sin (2 \theta) \cos (\omega t), \\
J_{x}^{\text {odd }}(t) & =-\frac{\omega}{A} \epsilon \sin \theta \sin (\omega t)\left[C_{0}+C_{2}+\cdots+D_{2}+D_{4}+\cdots\right]= \\
& =t_{z x}^{\text {odd }}\left(\hat{\boldsymbol{M}}=\hat{\boldsymbol{e}}_{x}\right) \frac{\omega}{A} \epsilon \sin \theta \sin (\omega t) .
\end{aligned}
$$

Even if $A_{2}, B_{2}, C_{2}$ and $D_{2}$ are non-zero, i.e., even in the presence of anisotropic SOT, the ISOT for magnetization precession around the $x$ axis can thus be expressed in terms of the torkance for magnetization along $x$. The even and odd contributions are again phase-shifted and the dependence on the cone angle is $\propto \sin \theta$ in the limit of small $\theta$ like in the case of magnetization precession around the $z$ axis, promising a significantly larger ISOT signal [39] compared to the case with magnetization precessing around the $y$ axis.

The main result of this subsection are the expressions for the ISOT currents given in Eq. (68) (magnetization precession around $z$ ), Eq. (71) (magnetization precession around $y$ ) and Eq. (74) (magnetization precession around $x)$. We stress that these expressions have been derived without any assumptions on the underlying mechanism (such as SHE or interfacial SOI) and are thus generally valid in bilayer systems with continuous rotational symmetry around the $z$ axis. In all three cases, the coefficients $C_{0}, C_{2}, \ldots$ and $D_{2}, D_{4}, \ldots$, which govern the odd torkance, give rise to an ac current, but never to a dc current. Thus, complete characterization of ISOT in experiments requires the measurement of the ac component.

\section{B. Reciprocity between the even SOT and the even ISOT}

In magnetic bilayer systems that involve a normal metal (NM) layer with large SHE it is expected that an important contribution to the even SOT arises from SHE [22, 23, 27, 52]. In particular when the NM layer is thin, the SHE in the NM layer will generally differ from the SHE in a corresponding bulk system. Even when the NM layer is thick, close to the interface with the magnet the electronic structure is modified due to the hybridization of the electronic states of the NM with those of the ferromagnet (FM). This electronic structure change is expected to entail a modification of the SHE in the NM close to the interface. Furthermore, the proximity with the FM layer induces magnetic moments in the NM at the interface due to which the SHE is also modified [53]. Additionally, qualitatively new mechanisms for SHE are added by the presence of the interface: When an electric field is applied to the bilayer in the in-plane direction, part of the in-plane electric current is carried by interface states that are evanescent waves along the stacking direction in the NM. That evanescent waves can also contribute to the SHE has been discussed [54] in the context of tunnel junctions but is also true for evanescent waves in all-metallic bilayer systems.

Rather than using the term SHE only for the bulk contribution, we will in this work often denote by SHE the total spin current generated by an applied electric field, including the interface modifications discussed above into the term SHE. Recently, we have shown within $a b$ initio calculations that the even SOT in $\mathrm{Co} / \mathrm{Pt}$ and $\mathrm{Mn} / \mathrm{W}$ bilayers arises from the flux of spin current from the NM into the FM layer [31]. Within our terminology this spin flux arises from the SHE.

In the following we discuss a minimal model to describe the SHE-contribution to the even SOT. We consider a bilayer system composed of a semi-infinite ferromagnetic layer (FM) on a semi-infinite normal metal (NM). The interface between FM and $\mathrm{NM}$ is located at $z=0$. We estimate the SOT arising from the SHE in NM, when an electric field $E_{x} \hat{\boldsymbol{e}}_{x}$ is applied in $x$ direction. Deep inside NM, i.e., for $z \ll 0$, the spin current density flowing in $z$ direction is

$$
Q_{y}=\sigma_{z x}^{y} E_{x}=\frac{\hbar}{2 e} \sigma_{x x} E_{x} \tan \gamma_{\mathrm{SHE}}
$$

where $\sigma_{z x}^{y}$ is the SHE conductivity in NM, $\tan \gamma_{\mathrm{SHE}}$ is the SHE angle and $\sigma_{x x}$ is the normal conductivity in NM. As discussed above the SHE is generally expected to be modified close to the NM/FM interface. However, in order to obtain a minimal model we neglect this expected position-dependence of SHE and assume that the SHE can effectively be described by a single parameter $\sigma_{z x}^{y}$. We assume that a fraction $\xi$ of $Q_{y}$ is transmitted through the NM/FM interface and absorbed by FM, thereby caus- 
ing a torque on its magnetization, which we assume to point in $z$ direction. This SHE-to-SOT efficiency $\xi$ can be thought of as the spin-current transmissivity [55] or transparency [56] of the NM/FM interface. In metallic magnetic bilayer systems $\xi$ is typically of the order of 1: In experiments on $\mathrm{NiFe} / \mathrm{Pt}$ it was estimated to be $\xi \approx 0.4-0.6$ 55]. In ab initio calculations of $\mathrm{FePt} / \mathrm{Pt}$ $\xi \approx 0.6$ was found [57]. Denoting the $x y$ cross sectional area of the unit cell by $A$, the torque per unit cell is given by $T_{y}^{\text {even }}=\xi A Q_{y}=t_{y x}^{\text {even }} E_{x}$ with

$$
t_{y x}^{\mathrm{even}}\left(\hat{\boldsymbol{M}}=\hat{\boldsymbol{e}}_{z}\right)=\xi A \sigma_{z x}^{y}=\xi A \frac{\hbar}{2 e} \sigma_{x x} \tan \gamma_{\mathrm{SHE}} .
$$

Next, we consider the even ISOT arising from the combined action of spin pumping and ISHE. The spin current density pumped adiabatically into NM is determined by [33]

$$
\boldsymbol{Q}(z=0)=\frac{\hbar}{4 \pi} \operatorname{Re} g^{\uparrow \downarrow} \hat{\boldsymbol{M}} \times \frac{d \hat{\boldsymbol{M}}}{d t},
$$

where $g^{\uparrow \downarrow}$ is the (generally complex) spin mixing conductance per cross-sectional area. The imaginary part of $g^{\uparrow \downarrow}$ is assumed to be negligible in Eq. (77). If spin transport in NM is diffusive, a spin accumulation $s(z)$ forms in NM due to the spins pumped into NM. The spin current in NM is proportional to the gradient of the spin accumulation $s(z)$. Since the spin accumulation decays exponentially in NM, $s(z)=s(0) e^{z / \lambda_{\mathrm{sd}}}$, where $\lambda_{\mathrm{sd}}$ is the spin diffusion length, also the spin current decays exponentially in NM, i.e., $\boldsymbol{Q}(z)=\boldsymbol{Q}(0) e^{z / \lambda_{\mathrm{sd}}}[34$, 39]. In the case of magnetization precession around the $y$ axis, Eq. (69), the dc spin current flowing in NM in $z$ direction is therefore given by

$$
Q_{y}(z)=-\frac{\hbar \omega}{4 \pi} \operatorname{Re} g^{\uparrow \downarrow} \sin ^{2}(\theta) \epsilon e^{z / \lambda_{\mathrm{sd}}} .
$$

Due to ISHE this spin current is converted into an inplane charge current flowing in $x$ direction:

$$
\begin{aligned}
j_{x}^{\text {even }}(z) & =-\frac{2 e}{\hbar} Q_{y}(z) \tan \gamma_{\mathrm{ISHE}}= \\
& =\frac{e \omega}{2 \pi} \operatorname{Re} g^{\uparrow \downarrow} \sin ^{2}(\theta) \epsilon e^{z / \lambda_{\mathrm{sd}}} \tan \gamma_{\mathrm{ISHE}}
\end{aligned}
$$

where $\tan \gamma_{\text {ISHE }}$ is the ISHE-angle. Thus, a single characteristic length, the spin diffusion length $\lambda_{\mathrm{sd}}$, determines the position dependence of $s(z), Q_{y}(z)$ and $j_{x}^{\text {even }}(z)$ within this model:

$$
j_{x}^{\text {even }}(z) \propto Q_{y}(z) \propto s(z) \propto e^{z / \lambda_{\mathrm{sd}}} .
$$

Integration of the current density Eq. (79) from $z=-\infty$ to $z=0$ yields the current per length flowing in NM:

$$
J_{x}^{\text {even }}=\frac{e \omega}{2 \pi} \operatorname{Re} g^{\uparrow \downarrow} \sin ^{2}(\theta) \epsilon \lambda_{\mathrm{Sd}} \tan \gamma_{\mathrm{ISHE}} .
$$

Using the small-cone limit of Eq. (70) and assuming $A_{2}=$ $B_{2}=A_{4}=\cdots=0$ we obtain the alternative expression

$$
J_{x}^{\text {even }}=\frac{\omega}{A} \sin ^{2}(\theta) \epsilon A_{0} .
$$

Equating the two expressions for $J_{x}^{\text {even }}$ yields

$$
A_{0}=A \frac{e}{2 \pi} \operatorname{Re} g^{\uparrow \downarrow} \lambda_{\text {sd }} \tan \gamma_{\text {ISHE }} .
$$

Application of $t_{y x}^{\text {even }}\left(\hat{\boldsymbol{M}}=\hat{\boldsymbol{e}}_{z}\right)=A_{0}$ leads to

$$
\lambda_{\mathrm{sd}}=\frac{2 \pi t_{y x}^{\text {even }}\left(\hat{\boldsymbol{M}}=\hat{\boldsymbol{e}}_{z}\right)}{e A \operatorname{Re} g^{\uparrow \downarrow} \tan \gamma_{\mathrm{ISHE}}} .
$$

Employing Eq. (76) and assuming $\tan \gamma_{\mathrm{ISHE}}=\tan \gamma_{\mathrm{SHE}}$ we can recast Eq. (84) as

$$
\lambda_{\mathrm{sd}}=\frac{\xi \hbar \pi \sigma_{x x}}{e^{2} \operatorname{Re} g^{\uparrow \downarrow}} .
$$

Eq. (84) relates the SHE-to-SOT efficiency $\xi$ with the parameters we use to model the ISOT current and thereby expresses the reciprocity between SOT and ISOT.

Even though this minimal model is derived for semiinfinite layers it can be applied to bilayers of finite thickness when the layer thickness is much larger than $\lambda_{\text {sd }}$. When NM has the finite thickness $D$, i.e., $-D \leq z \leq 0$, and when $D \gg \lambda_{\text {sd }}$ is not satisfied, Eq. (78) needs to be replaced by [34]

$$
Q_{y}(z)=-\frac{\hbar \omega}{4 \pi} \operatorname{Re} g^{\uparrow \downarrow} \sin ^{2}(\theta) \epsilon \frac{\sinh \frac{z+D}{\lambda_{\mathrm{sd}}}}{\sinh \frac{D}{\lambda_{\mathrm{sd}}}},
$$

in order to take into account that the spin current is reflected at the boundary of $\mathrm{NM}$ at $z=-D$.

In Sec. IVB and IVC we will compare ISOT current and spin current densities obtained from ab initio calculations to the minimal model described above. We will show that the minimal model provides a satisfactory description of the $a b$ initio results. We will discuss that the main shortcoming of the minimal model is the assumption that SHE and ISHE can be described by a single position-independent parameter, whereby the modification of SHE and ISHE close to the interface is neglected.

\section{Reciprocity between the odd SOT and the odd ISOT}

In Sec. II we demonstrated the reciprocity between ISOT and SOT on general grounds. The odd SOT in the bilayer systems considered in this work arises dominantly from the intraband contribution to Eq. (8). This intraband contribution can also be obtained from Boltzmann transport theory within the constant relaxation time approximation. In this subsection we study the odd SOT 
and the odd ISOT within Boltzmann transport theory and show that the obtained expressions satisfy the reciprocity formulated previously in Sec. II

When an electric field $\boldsymbol{E}$ is applied the occupation number $f_{\boldsymbol{k} n}$ of band $n$ at $k$-point $\boldsymbol{k}$ changes according to

$$
\delta f_{\boldsymbol{k} n}^{(1)}=-e \tau \boldsymbol{v}_{\boldsymbol{k} n} \cdot \boldsymbol{E} \delta\left(\mathcal{E}_{\mathrm{F}}-\mathcal{E}_{\boldsymbol{k} n}\right),
$$

where $\tau$ is the relaxation time, $\mathcal{E}_{\mathrm{F}}$ is the Fermi energy and $\boldsymbol{v}_{\boldsymbol{k} n}=\left\langle\psi_{\boldsymbol{k} n}|\boldsymbol{v}| \psi_{\boldsymbol{k} n}\right\rangle$ is the group velocity of band $n$ at $k$-point $\boldsymbol{k}$. The change $\delta f_{\boldsymbol{k n}}^{(1)}$ of the occupancies results in the contribution

$$
\begin{aligned}
j_{\alpha}^{(1)} & =-\frac{e}{V \mathcal{N}} \sum_{\boldsymbol{k} n} v_{\boldsymbol{k} \alpha n} \delta f_{\boldsymbol{k} n}^{(1)} \\
& =\frac{e^{2} \tau}{V \mathcal{N}} \sum_{\boldsymbol{k} n \beta} v_{\boldsymbol{k} \alpha n} v_{\boldsymbol{k} \beta n} E_{\beta} \delta\left(\mathcal{E}_{\mathrm{F}}-\mathcal{E}_{\boldsymbol{k} n}\right)
\end{aligned}
$$

to the electric current density and in the contribution

$$
\begin{aligned}
T_{\alpha}^{(1)} & =-\sum_{\boldsymbol{k n}} \mathcal{T}_{\boldsymbol{k} \alpha n} \delta f_{\boldsymbol{k} n}^{(1)} \\
& =\frac{e \tau}{\mathcal{N}} \sum_{\boldsymbol{k} n \beta} \mathcal{T}_{\boldsymbol{k} \alpha n} v_{\boldsymbol{k} \beta n} E_{\beta} \delta\left(\mathcal{E}_{\mathrm{F}}-\mathcal{E}_{\boldsymbol{k} n}\right),
\end{aligned}
$$

to the torque, where $v_{\boldsymbol{k} \alpha n}$ and $\mathcal{T}_{\boldsymbol{k} \alpha n}$ are the $\alpha$-th Cartesian components of the group velocity $\boldsymbol{v}_{\boldsymbol{k} n}$ and of the torque $\mathcal{T}_{\boldsymbol{k} n}=\left\langle\psi_{\boldsymbol{k} n}|\mathcal{T}| \psi_{\boldsymbol{k} n}\right\rangle$, respectively.

When the system is perturbed not by an electric field but by the time-dependence of the magnetization direction $\hat{M}(t)$ the change of the occupancies is given by

$$
\delta f_{\boldsymbol{k} n}^{(2)}=\tau \delta\left(\mathcal{E}_{\mathrm{F}}-\mathcal{E}_{\boldsymbol{k} n}\right) \mathcal{T}_{\boldsymbol{k} n} \cdot\left[\hat{\boldsymbol{M}}(t) \times \frac{d \hat{\boldsymbol{M}}(t)}{d t}\right]
$$

instead of Eq. (87). Eq. (90) follows from

$$
\begin{aligned}
& \frac{\delta f_{\boldsymbol{k} n}^{(2)}}{\tau}=-\frac{\partial f_{\boldsymbol{k} n}}{\partial \hat{\boldsymbol{M}}} \cdot \frac{d \hat{\boldsymbol{M}}}{d t}=-\frac{\partial f_{\boldsymbol{k} n}}{\partial \mathcal{E}_{\boldsymbol{k} n}} \frac{\partial \mathcal{E}_{\boldsymbol{k} n}}{\partial \hat{\boldsymbol{M}}} \cdot \frac{d \hat{\boldsymbol{M}}}{d t}= \\
& =\delta\left(\mathcal{E}_{\mathrm{F}}-\mathcal{E}_{\boldsymbol{k} n}\right)\left[\hat{\boldsymbol{M}}(t) \times \frac{\partial \mathcal{E}_{\boldsymbol{k} n}}{\partial \hat{\boldsymbol{M}}}\right] \cdot\left[\hat{\boldsymbol{M}}(t) \times \frac{d \hat{\boldsymbol{M}}(t)}{d t}\right] \\
& =\delta\left(\mathcal{E}_{\mathrm{F}}-\mathcal{E}_{\boldsymbol{k} n}\right) \boldsymbol{\mathcal { T }}_{\boldsymbol{k} n} \cdot\left[\hat{\boldsymbol{M}}(t) \times \frac{d \hat{\boldsymbol{M}}(t)}{d t}\right],
\end{aligned}
$$

where we set the temperature in the Fermi-Dirac distribution function to zero such that $f_{\boldsymbol{k} n}=\theta\left(\mathcal{E}_{\mathrm{F}}-\mathcal{E}_{\boldsymbol{k} n}\right)$ and $\partial f_{\boldsymbol{k} n} / \partial \mathcal{E}_{\boldsymbol{k} n}=-\delta\left(\mathcal{E}_{\mathrm{F}}-\mathcal{E}_{\boldsymbol{k} n}\right)$. Additionally, we made use of

$$
\left[\hat{\boldsymbol{M}}(t) \times \frac{\partial \mathcal{E}_{\boldsymbol{k} n}}{\partial \hat{\boldsymbol{M}}}\right]=\hat{\boldsymbol{M}}(t) \times\left\langle\psi_{\boldsymbol{k} n}\left|\frac{\partial H_{\hat{\boldsymbol{M}}}}{\partial \hat{\boldsymbol{M}}}\right| \psi_{\boldsymbol{k} n}\right\rangle=\boldsymbol{\mathcal { T }}_{\boldsymbol{k} n} .
$$

Eq. (90) and Eq. (91) hold under the condition that the frequency $\omega$ of the precession of magnetization is small compared to the relaxation rate $\tau^{-1}$, i.e., $\omega \ll \tau^{-1}$. If the condition $\omega \ll \tau^{-1}$ is violated one needs to solve the Boltzmann equation assuming an explicit timedependence of the distribution function. The expressions valid in that case are obtained by replacing $\tau$ in Eq. (90) and Eq. (91) as follows:

$$
\tau \rightarrow \frac{\tau}{1-i \omega \tau}
$$

For magnetic bilayers such as $\mathrm{Co} / \mathrm{Pt}$ we estimate that $10 \mathrm{THz}<1 /(2 \pi \tau)$, which is much larger than ferromagnetic resonance frequencies in the $\mathrm{GHz}$ range. Therefore, we will always assume $\omega \ll \tau^{-1}$ in the following.

The current density induced due to the timedependence of magnetization can be obtained from the change of occupancies $\delta f_{\boldsymbol{k n}}^{(2)}$ given in Eq. (90):

$$
\begin{aligned}
j_{\alpha}^{(2)} & =-\frac{e}{V \mathcal{N}} \sum_{\boldsymbol{k} n} v_{\boldsymbol{k} \alpha n} \delta f_{\boldsymbol{k} n}^{(2)} \\
& =-\frac{e \tau}{V \mathcal{N}} \sum_{\boldsymbol{k} n} v_{\boldsymbol{k} \alpha n} \delta\left(\mathcal{E}_{\mathrm{F}}-\mathcal{E}_{\boldsymbol{k} n}\right) \mathcal{T}_{\boldsymbol{k} n} \cdot\left[\hat{\boldsymbol{M}}(t) \times \frac{d \hat{\boldsymbol{M}}(t)}{d t}\right] .
\end{aligned}
$$

Similarly, the torque which damps the magnetization dynamics is given by

$$
\begin{aligned}
& T_{\alpha}^{(2)}=-\frac{1}{\mathcal{N}} \sum_{\boldsymbol{k} n} \mathcal{T}_{\boldsymbol{k} \alpha n} \delta f_{\boldsymbol{k} n}^{(2)}= \\
& =-\frac{\tau}{\mathcal{N}} \sum_{\boldsymbol{k} n} \mathcal{T}_{\boldsymbol{k} \alpha n} \delta\left(\mathcal{E}_{\mathrm{F}}-\mathcal{E}_{\boldsymbol{k} n}\right) \mathcal{T}_{\boldsymbol{k} n} \cdot\left[\hat{\boldsymbol{M}}(t) \times \frac{d \hat{\boldsymbol{M}}(t)}{d t}\right] .
\end{aligned}
$$

We can combine Eq. (88), Eq. (89), Eq. (94) and Eq. (95) in the form of Eq. (22) as follows:

$$
\left(\begin{array}{c}
\tilde{j} \\
\tilde{\boldsymbol{T}} / V
\end{array}\right)=\left(\begin{array}{cc}
\tilde{\boldsymbol{\sigma}} & -\tilde{\boldsymbol{t}}^{\mathrm{T}} / V \\
\tilde{\boldsymbol{t}} / V & -\tilde{\boldsymbol{\Lambda}}
\end{array}\right)\left(\begin{array}{c}
\boldsymbol{E} \\
\hat{\boldsymbol{M}} \times \frac{d \hat{\boldsymbol{M}}}{d t}
\end{array}\right),
$$

where we defined $\tilde{\boldsymbol{j}}=\boldsymbol{j}^{(1)}+\boldsymbol{j}^{(2)}$ and $\tilde{\boldsymbol{T}}=\boldsymbol{T}^{(1)}+\boldsymbol{T}^{(2)}$. We use the tilde to recall that according to Eq. (88), Eq. (89), Eq. (94) and Eq. (95) only intraband terms are considered in $\tilde{\boldsymbol{j}}$ and $\tilde{\boldsymbol{T}}$, while the complete expression for current density and torque contains additional interband terms. The linear response coefficients $\tilde{\boldsymbol{\sigma}}, \tilde{\boldsymbol{t}}$ and $\tilde{\boldsymbol{\Lambda}}$ are given by

$$
\begin{aligned}
\tilde{\sigma}_{\alpha \beta} & =\frac{e^{2} \tau}{V \mathcal{N}} \sum_{\boldsymbol{k} n} v_{\boldsymbol{k} \alpha n} v_{\boldsymbol{k} \beta n} \delta\left(\mathcal{E}_{\mathrm{F}}-\mathcal{E}_{\boldsymbol{k} n}\right), \\
\tilde{t}_{\alpha \beta} & =\frac{e \tau}{\mathcal{N}} \sum_{\boldsymbol{k} n} \mathcal{T}_{\boldsymbol{k} \alpha n} v_{\boldsymbol{k} \beta n} \delta\left(\mathcal{E}_{\mathrm{F}}-\mathcal{E}_{\boldsymbol{k} n}\right), \\
\tilde{\Lambda}_{\alpha \beta} & =\frac{\tau}{V \mathcal{N}} \sum_{\boldsymbol{k} n} \mathcal{T}_{\boldsymbol{k} \alpha n} \mathcal{T}_{\boldsymbol{k} \beta n} \delta\left(\mathcal{E}_{\mathrm{F}}-\mathcal{E}_{\boldsymbol{k} n}\right) .
\end{aligned}
$$

$\tilde{\boldsymbol{\sigma}}$ and $\tilde{\boldsymbol{\Lambda}}$ are even with respect to reversal of magnetization direction $\hat{\boldsymbol{M}}$, while $\tilde{\boldsymbol{t}}$ is odd. Eq. (96) clearly shows that the tensor $\tilde{\boldsymbol{t}}$ governs both the odd SOT and the 
odd ISOT. The Gilbert damping $\tilde{\boldsymbol{\alpha}}$ is related to $\tilde{\boldsymbol{\Lambda}}$ by $\tilde{\boldsymbol{\alpha}}=|\gamma| \tilde{\boldsymbol{\Lambda}} /\left(\mu_{0} M\right)$ (see Eq. (20) $)$, i.e.,

$$
\tilde{\alpha}_{\alpha \beta}=\frac{|\gamma| \tau}{\mu_{0} V M \mathcal{N}} \sum_{\boldsymbol{k} n} \mathcal{T}_{\boldsymbol{k} \alpha n} \mathcal{T}_{\boldsymbol{k} \beta n} \delta\left(\mathcal{E}_{\mathrm{F}}-\mathcal{E}_{\boldsymbol{k} n}\right)
$$

which agrees with the intraband term in the torquecorrelation formula of the Gilbert damping [50, 58, 59]. Thus, Eq. (90) leads to a coherent description of the intraband contributions to both the Gilbert damping and the odd ISOT. Moreover, the expression obtained for the odd ISOT is reciprocal to the odd direct SOT.

\section{FIRST PRINCIPLES CALCULATIONS}

\section{A. Computational method}

In the following we will discuss SOTs and ISOTs for a bilayer composed of 3 layers of hcp Co on 20 layers of fcc $\mathrm{Pt}(111)$, denoted in the following as $\mathrm{Co}(3) / \mathrm{Pt}(20)$. We label the atomic layers of the Pt layer by Pt1 through Pt20, where Pt20 is at the Co/Pt interface. Likewise, we label the atomic layers of the Co layer by Co1 through $\mathrm{Co} 3$, where $\mathrm{Co} 1$ is at the $\mathrm{Co} / \mathrm{Pt}$ interface. We introduce a cartesian coordinate system such that the $z$ axis is perpendicular to the atomic layers, i.e., along the out-ofplane direction, and Pt20 has a smaller $z$ coordinate than Co1. The magnetization direction is set to $\hat{M}=\hat{\boldsymbol{e}}_{z}$ in the calculation. In order to perform the linear-response calculations of the torkance computationally efficiently, the Wannier interpolation technique is employed [60 62]. For this purpose we express the electronic structure in terms of maximally localized Wannier functions (MLWFs), using 18 MLWFs per atom. Details of the electronic structure calculation of $\mathrm{Co}(3) / \mathrm{Pt}(20)$ are given in Ref. 31.

Within the independent particle approximation the torkance $\boldsymbol{t}$ defined in Eq. (8) can be expressed as sum of three terms, $t_{\alpha \beta}=t_{\alpha \beta}^{\mathrm{I}(\mathrm{a})}+t_{\alpha \beta}^{\mathrm{I}(\mathrm{b})}+t_{\alpha \beta}^{\mathrm{II}}$, where [31, 45]

$$
\begin{aligned}
& t_{\alpha \beta}^{\mathrm{I}(\mathrm{a})}= \frac{e}{\mathcal{N} h} \sum_{\boldsymbol{k}} \operatorname{Tr}\left\langle\mathcal{T}_{\alpha} G_{\boldsymbol{k}}^{\mathrm{R}}\left(\mathcal{E}_{\mathrm{F}}\right) v_{\beta} G_{\boldsymbol{k}}^{\mathrm{A}}\left(\mathcal{E}_{\mathrm{F}}\right)\right\rangle \\
& t_{\alpha \beta}^{\mathrm{I}(\mathrm{b})}=-\frac{e}{\mathcal{N} h} \sum_{\boldsymbol{k}} \operatorname{Re} \operatorname{Tr}\left\langle\mathcal{T}_{\alpha} G_{\boldsymbol{k}}^{\mathrm{R}}\left(\mathcal{E}_{\mathrm{F}}\right) v_{\beta} G_{\boldsymbol{k}}^{\mathrm{R}}\left(\mathcal{E}_{\mathrm{F}}\right)\right\rangle \\
& t_{\alpha \beta}^{\mathrm{II}}=\frac{e}{\mathcal{N} h} \sum_{\boldsymbol{k}} \int_{-\infty}^{\mathcal{E}_{\mathrm{F}}} d \mathcal{E} \operatorname{Re} \operatorname{Tr}\left\langle\mathcal{T}_{\alpha} G_{\boldsymbol{k}}^{\mathrm{R}}(\mathcal{E}) v_{\beta} \frac{d G_{\boldsymbol{k}}^{\mathrm{R}}(\mathcal{E})}{d \mathcal{E}}\right. \\
&\left.\quad-\mathcal{T}_{\alpha} \frac{d G_{\boldsymbol{k}}^{\mathrm{R}}(\mathcal{E})}{d \mathcal{E}} v_{\beta} G_{\boldsymbol{k}}^{\mathrm{R}}(\mathcal{E})\right\rangle,
\end{aligned}
$$

with $G_{\boldsymbol{k}}^{\mathrm{R}}(\mathcal{E})$ the retarded Green function at $k$ point $\boldsymbol{k}$ and energy $\mathcal{E}, G_{\boldsymbol{k}}^{\mathrm{A}}(\mathcal{E})$ the advanced one, $\mathcal{N}$ the number of $k$ points and $\mathcal{E}_{\mathrm{F}}$ the Fermi energy. We model the effect of disorder by a phenomenological band broadening $\Gamma$ in the Green functions, i.e., $G_{\boldsymbol{k}}^{\mathrm{R}}(\mathcal{E})=\hbar\left[\mathcal{E}-H_{\boldsymbol{k}}+i \Gamma\right]^{-1}$.
We discuss the direct SOT in terms of the torkance, which we compute according to Eq. (99). In order to obtain atom-resolved torkances, we replace the torque operator in Eq. (99) by an atom-resolved torque operator (see Ref. [31] for details). We calculate the induced ISOT current in the $\mathrm{Co}(3) / \mathrm{Pt}(20)$ bilayer using Eq. (64) and the torkance obtained from Eq. (99). However, it is desirable to determine also the spatial profile of the ISOT current along the $z$ direction. For this purpose we define the layer-resolved velocity operator

$$
v_{\boldsymbol{k} \alpha n m}(L)=v_{\boldsymbol{k} \alpha n m} \theta_{n}(L) \theta_{m}(L),
$$

where $\theta_{m}(L)=1$ if MLWF orbital $m$ belongs to layer $L$ and zero otherwise. Here, each MLWF is attributed to the one atomic layer in which the center of the MLWF is located and

$$
v_{\boldsymbol{k} \alpha n m}=\frac{1}{\hbar} \sum_{\boldsymbol{R}} e^{i \boldsymbol{k} \cdot \boldsymbol{R}_{i \mathrm{R}_{\alpha}}\left\langle W_{n \mathbf{0}}|H| W_{m \boldsymbol{R}}\right\rangle}
$$

is the $\alpha$-th cartesian component of the velocity operator at $k$ point $\boldsymbol{k}$ expressed in the basis of Wannier functions. Replacing $v_{\alpha}$ in Eq. (99) by $v_{\alpha}(L)$ allows us to compute the ISOT current within the atomic layer $L$.

The direct SOT is a response to the applied electric field $\boldsymbol{E}$, which exerts the mechanical force $-e \boldsymbol{E}$ on the electrons. By artificially switching off the force $-e \boldsymbol{E}$ for some atomic layers, we investigate which atomic layers participate in generating the SOT. Noting that the mechanical force is represented in Eq. (99) by the velocity operator, we replace $v_{\alpha}$ in Eq. (99) by $v_{\alpha}(L)$ in order to study the SOT generated when the force $-e \boldsymbol{E}$ acts only on the electrons in the atomic layer $L$. Thus, the replacement of $v_{\alpha}$ by $v_{\alpha}(L)$ in Eq. (99) provides us not only with the information on how the ISOT current is distributed in $\mathrm{Co}(3) / \mathrm{Pt}(20)$ along the stacking direction, but additionally it also provides us with the information in which atomic layers the action of the force $-e \boldsymbol{E}$ is essential for the direct SOT. This results from the reciprocity between ISOT and SOT, which implies that the atomic layers that carry the ISOT current agree to the atomic layers that participate in generating the SOT. In order to describe the situation where the mechanical force is switched off for the atomic layers Pt1 through $L-1$ we use the sum of Eq. (100) for the layers $L, L+1, \ldots$, i.e., we use the modified velocity operator

$$
\bar{v}_{\boldsymbol{k} \alpha n m}(L)=v_{\boldsymbol{k} \alpha n m} \sum_{L_{1} \geq L} \sum_{L_{2} \geq L} \theta_{n}\left(L_{1}\right) \theta_{m}\left(L_{2}\right)
$$

in Eq. (99). Here, the functions $\theta_{m}(L)$ are defined like above, below Eq. (100).

As discussed in Sec. IIIB the spin current flowing in $z$ direction mediates an important contribution to the even ISOT in bilayer systems. Thus, it is desirable to determine its spatial profile along the $z$ direction. For this 
purpose, we define the layer-resolved spin current density operator $\mathcal{Q}_{s}(L)$ for spin currents flowing in $z$ direction by

$$
\left\langle\psi_{\boldsymbol{k} n}\left|\mathcal{Q}_{s}(L)\right| \psi_{\boldsymbol{k} m}\right\rangle=\frac{1}{A} \int_{S_{L}} d \boldsymbol{S} \cdot\left\langle\psi_{\boldsymbol{k} n}\left|\mathcal{Q}_{s}(\boldsymbol{r})\right| \psi_{\boldsymbol{k} m}\right\rangle,
$$

where the integration is over the boundary $S_{L}$ between layers $L-1$ and $L, A$ is the $x y$ cross sectional area of the unit cell, and $\mathcal{Q}_{s}(\boldsymbol{r})$ is the spin current density operator at point $\boldsymbol{r}$. SOI is only strong close to the atomic nuclei, because it is proportional to the electrostatic potential gradient. Since the boundary $S_{L}$ is chosen to lie in the interstitial region, where SOI is negligible, the nonrelativistic spin current density operator can be used:

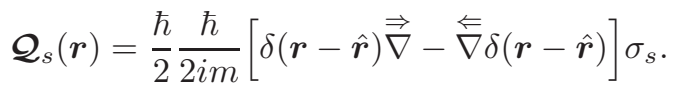

By replacing in Eq. (5) the current density operator $-e v_{\alpha} / V$ by $\mathcal{Q}_{s}(L)$, we can determine the spin current profile along the stacking direction of the $\mathrm{Co}(3) / \mathrm{Pt}(20)$ bilayer:

$$
Q_{s}(L, t)=\frac{1}{A} \sum_{\beta} w_{s \beta}(L, \hat{\boldsymbol{M}}(t))\left[\hat{\boldsymbol{M}}(t) \times \frac{d \hat{\boldsymbol{M}}(t)}{d t}\right]_{\beta},
$$

where we defined

$$
w_{s \beta}(L, \hat{\boldsymbol{M}})=-A \lim _{\omega \rightarrow 0} \frac{\operatorname{Im} G_{\mathcal{Q}_{s}(L), \mathcal{T}_{\beta}}^{\mathrm{R}}(\hbar \omega, \hat{\boldsymbol{M}})}{\hbar \omega},
$$

with the Fourier transform of the retarded spin-current torque correlation function

$$
G_{\mathcal{Q}_{s}(L), \mathcal{T}_{\beta}}^{\mathrm{R}}(\hbar \omega, \hat{\boldsymbol{M}})=-i \int_{0}^{\infty} d t e^{i \omega t}\left\langle\left[\mathcal{Q}_{s}(L), \mathcal{T}_{\beta}(-t)\right]_{-}\right\rangle .
$$

Within the independent particle approximation Eq. (106) becomes $w_{s \beta}(L)=w_{s \beta}^{\mathrm{I}(\mathrm{a})}(L)+w_{s \beta}^{\mathrm{I}(\mathrm{b})}(L)+w_{s \beta}^{\mathrm{II}}(L)$, with

$$
\begin{aligned}
w_{s \beta}^{\mathrm{I}(\mathrm{a})}(L) & =\frac{e A}{\mathcal{N} h} \sum_{\boldsymbol{k}} \operatorname{Tr}\left\langle\mathcal{Q}_{s}(L) G_{\boldsymbol{k}}^{\mathrm{R}}\left(\mathcal{E}_{\mathrm{F}}\right) \mathcal{T}_{\beta} G_{\boldsymbol{k}}^{\mathrm{A}}\left(\mathcal{E}_{\mathrm{F}}\right)\right\rangle \\
w_{s \beta}^{\mathrm{I}(\mathrm{b})}(L) & =-\frac{e A}{\mathcal{N} h} \sum_{\boldsymbol{k}} \operatorname{Re} \operatorname{Tr}\left\langle\mathcal{Q}_{s}(L) G_{\boldsymbol{k}}^{\mathrm{R}}\left(\mathcal{E}_{\mathrm{F}}\right) \mathcal{T}_{\beta} G_{\boldsymbol{k}}^{\mathrm{R}}\left(\mathcal{E}_{\mathrm{F}}\right)\right\rangle \\
w_{s \beta}^{\mathrm{II}}(L) & =\frac{e A}{\mathcal{N} h} \sum_{\boldsymbol{k}} \int_{-\infty}^{\mathcal{E}_{\mathrm{F}}} d \mathcal{E} \operatorname{Re} \operatorname{Tr}\left\langle\mathcal{Q}_{s}(L) G_{\boldsymbol{k}}^{\mathrm{R}}(\mathcal{E}) \mathcal{T}_{\beta} \frac{d G_{\boldsymbol{k}}^{\mathrm{R}}(\mathcal{E})}{d \mathcal{E}}\right. \\
& \left.-\mathcal{Q}_{s}(L) \frac{d G_{\boldsymbol{k}}^{\mathrm{R}}(\mathcal{E})}{d \mathcal{E}} \mathcal{T}_{\beta} G_{\boldsymbol{k}}^{\mathrm{R}}(\mathcal{E})\right\rangle,
\end{aligned}
$$

where we suppressed the $\hat{\boldsymbol{M}}$ dependence for notational convenience. Comparison of Eq. (77) and Eq. (105) yields the following expression for the spin mixing conductance:

$$
\operatorname{Re} g^{\uparrow \downarrow}=\frac{4 \pi}{\hbar A} w_{y y}(L=\mathrm{Co} 1),
$$

where $w_{y y}(L=\mathrm{Co} 1)$ is proportional to spin current flowing between the layers Pt20 and Co1. In Co/Pt bilayers $t_{y x}^{\text {even }}$ arises almost entirely from the spin-flux into the Colayer [31]. The extraction of Re $g^{\uparrow \downarrow}$ from $w_{y y}$ is therefore meaningful in this case despite the presence of SOI in the calculation.

Similarly, as discussed in Sec. IIIB, SHE provides an important contribution to the even SOT in bilayer systems. The spin currents of the direct SHE are generated by the applied electric field rather than by spin pumping. In order to investigate the layer-resolved spin current profile of these spin currents in $\mathrm{Co}(3) / \mathrm{Pt}(20)$, we define the coefficients

$$
q_{s \beta}(L, \hat{\boldsymbol{M}})=A e \lim _{\omega \rightarrow 0} \frac{\operatorname{Im} G_{\mathcal{Q}_{s}(L), v_{\beta}}^{\mathrm{R}}(\hbar \omega, \hat{\boldsymbol{M}})}{\hbar \omega} .
$$

For example, $q_{y x}(L)$ quantifies the linear response of spin currents flowing in $z$ direction with spin pointing in $y$ direction to the electric field in $x$ direction. Within the independent particle approximation $q_{s \beta}(L)$ is expressed similarly to the torkance (Eq. (99)): Only $\mathcal{T}_{\alpha}$ has to be replaced by $-A Q_{s}(L)$ in the expressions.

For a given atomic layer, the difference between spincurrent flowing in and spin-current flowing out is the spin-flux into that atomic layer. In $\mathrm{Co} / \mathrm{Pt}$ bilayer systems, the even SOT arises dominantly from the spin-flux into the Co layer [31]. The linear-response coefficient of spin flux into layer $L$ is given by

$$
\Delta q_{y x}(L)=q_{y x}(L)-q_{y x}(L+1),
$$

where according to Eq. (103) and Eq. (110) $q_{y x}(L)$ describes spin current flowing between layers $L-1$ and $L$ towards layer $L$ and $-q_{y x}(L+1)$ describes spin current flowing between layers $L$ and $L+1$ towards layer $L$.

\section{B. Even SOT}

We first discuss the even torkance $t_{y x, 25 \mathrm{meV}}^{\mathrm{even}}$ determined from Eq. (99). At $\Gamma=25 \mathrm{meV}$ we obtain $t_{y x, 25 \mathrm{meV}}^{\text {even }}=0.68 e a_{0}$ per unit cell, where $e a_{0}$ is the atomic unit of torkance, which amounts to $e a_{0}=8.478$. $10^{-30} \mathrm{Cm}$. A slightly smaller value of $t_{y x, 100 \mathrm{meV}}^{\text {even }}=$ $0.53 e a_{0}$ is calculated at $\Gamma=100 \mathrm{meV}$. Dividing these torkances by the magnetic moment per unit cell of $\mu=5.78 \mu_{\mathrm{B}}$ we compute the effective fields per applied electric field of $t_{y x, 25 \mathrm{meV}}^{\text {even }} / \mu=0.011 \mathrm{mTcm} / \mathrm{V}$ and $t_{y x, 100 \mathrm{meV}}^{\mathrm{even}} / \mu=0.0084 \mathrm{mTcm} / \mathrm{V}$.

In Fig. (11) we show the layer-resolved even torkance, i.e., the linear-response coefficient of the torque acting on the magnetization of a given layer, and the linearresponse coefficient of spin flux into layer $L$ (Eq. (111)). For the Co layers, layer-resolved torkances and spin fluxes coincide approximately. Thus, the even torkance in $\mathrm{Co}(3) / \mathrm{Pt}(20)$ arises dominantly from the spin current 


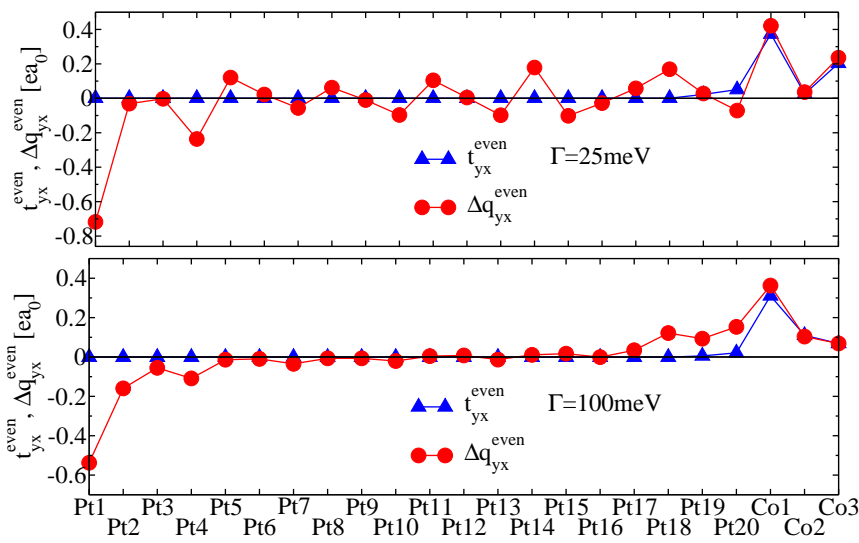

FIG. 1: Triangles: Layer-resolved even torkance $t_{y x}^{\text {even }}$ for broadenings of $\Gamma=25 \mathrm{meV}$ (upper graph) and $\Gamma=100 \mathrm{meV}$ (lower graph). Circles: Linear response coefficient of the layer-resolved spin flux $\Delta q_{y x}^{\text {even }}$, Eq. (111). Solid lines serve as guide to the eye.

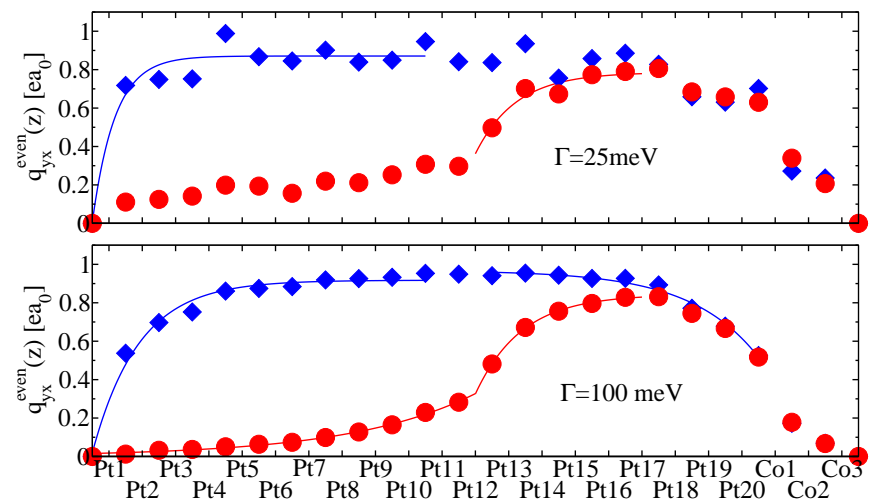

FIG. 2: Diamonds: Linear response coefficients $q_{y x}^{\text {even }}(z)$ of the layer-resolved spin current for $\Gamma=25 \mathrm{meV}$ (upper graph) and $\Gamma=100 \mathrm{meV}$ (lower graph). Circles: Linear response coefficients $q_{y x}^{\text {even }}(z)$ but with the mechanical force switched off for layers Pt1 through Pt12. Solid lines: Exponential fits according to Eq. (112), Eq. (113), Eq. (115) and Eq. (114).

flowing into the Co layer, consistent with the discussion in Sec. IIIB and with previous work on $\mathrm{Co} / \mathrm{Pt}$ bilayer systems 31].

In Fig. 2 we show the linear response coefficients of the layer-resolved spin current $q_{y x}^{\text {even }}(L)$ as diamonds for two values of broadening, $\Gamma=25 \mathrm{meV}$ and $\Gamma=100 \mathrm{meV}$ (see Eq. (110) for the definition of $q_{y x}^{\text {even }}(L)$ ). Evaluating the SHE-to-SOT conversion efficiency defined in Eq. (76) from the ratio of torkance to maximal spin current we obtain $\xi_{25 \mathrm{meV}}=t_{y x}^{\text {even }} /\left[q_{y x}^{\text {even }}(L=\right.$ Pt11 $\left.)\right]=0.74$. At $\Gamma=100 \mathrm{meV}$ the value is slightly lower: $\xi_{100 \mathrm{meV}}=0.57$. These values of $\xi$ resemble the experimentally determined spin-current transmissivities in Pt-based magnetic bilayer systems [55].

Computing the electric conductivities based on the same formalism as used for SOT and ISOT, we obtain $\sigma_{x x}^{25 \mathrm{meV}}=1.26 \cdot 10^{7} \mathrm{~S} / \mathrm{m}$ and $\sigma_{x x}^{100 \mathrm{meV}}=0.34 \cdot 10^{7} \mathrm{~S} / \mathrm{m}$. From these conductivities and the spin currents at the center of Pt, which are given by $q_{y x}^{\text {even }}(L=\mathrm{Pt} 11)$, we obtain the following SHE angles: $\tan \gamma_{\mathrm{SHE}}^{25 \mathrm{meV}}=0.029$ and $\tan \gamma_{\mathrm{SHE}}^{100 \mathrm{meV}}=0.109$. The SHE angle increases thus by a factor of 3.8 as $\Gamma$ is increased from $25 \mathrm{meV}$ to $100 \mathrm{meV}$. This increase of the SHE angle with increasing disorder is expected for the intrinsic SHE, because the intrinsic SHE conductivity $\sigma_{z x}^{y}$ (see Eq. (75)) depends only weakly on disorder, while the normal conductivity $\sigma_{x x}$ decreases with disorder. Indeed, the increase of the SHE angle by the factor of 3.8 is well explained by the ratio $\sigma_{x x}^{25 \mathrm{meV}} / \sigma_{x x}^{100 \mathrm{meV}}=3.7$.

At $\Gamma=100 \mathrm{meV}$ the line of blue diamonds illustrating $q_{y x}^{\text {even }}(z)$ in Fig. 2 is constant in the central region between Pt5 and Pt15, because the primary spin current generated by $\mathrm{SHE}$ is constant in this region and because secondary spin currents arising from the reflections of spin current at the surfaces and interfaces decay strongly spatially and therefore do not reach the central region between Pt5 and Pt15. One reason for the suppression of the spin current-profile in the region between Pt1 and Pt5 and in the region between Pt15 and Pt20 is the interference of the primary spin current from the SHE with secondary spin current reflected respectively from the surface and the interface. Additionally, as discussed in Sec. IIIB, we expect that the primary spin current generated by the SHE is itself dependent on position in these two regions and not constant like in the central region. In particular for the higher broadening of $\Gamma=100 \mathrm{meV}$ the spin current profiles from our ab initio calculations shown in Fig. 2 exhibit exponential behavior in the region Pt1 through Pt5 and in the region Pt15 through Pt20. At $\Gamma=100 \mathrm{meV}$ the spin current in the region between $\mathrm{Pt} 12$ and $\mathrm{Co} 1$ is well described by the exponential fit

$$
q_{y x}^{\text {even }}(z)=\left[0.97-0.35 e^{\left(z-z_{\mathrm{Pt} 20}\right) / \lambda_{\mathrm{SOT}, 3}^{100 \mathrm{meV}}}\right] e a_{0},
$$

where $z_{\mathrm{Pt} 20}$ is the $z$ coordinate of layer Pt20 and $\lambda_{\mathrm{SOT}, 3}^{100 \mathrm{meV}}=0.46 \mathrm{~nm}$. In the region from Pt1 to Pt10 the spin current is approximately given by

$$
q_{y x}^{\text {even }}(z)=\left[0.92-0.63 e^{-\left(z-z_{\mathrm{Pt} 1}\right) / \lambda_{\mathrm{SOT}, 4}^{100 \mathrm{meV}}}\right] e a_{0}
$$

with $\lambda_{\mathrm{SOT}, 4}^{100 \mathrm{meV}}=0.32 \mathrm{~nm}$. At the smaller broadening of $\Gamma=25 \mathrm{meV}$ we find $\lambda_{\mathrm{SOT}, 4}^{25 \mathrm{meV}}=0.15 \mathrm{~nm}$, but due to oscillations the first principles data are less well described by the exponential fit.

The length $\lambda_{\mathrm{SOT}, 4}^{100 \mathrm{meV}}$ describes the decay of spin current close to the vacuum boundary at Pt1, while the length $\lambda_{\mathrm{SOT}, 3}^{100 \mathrm{meV}}$ describes the decay of spin current close to the Co layer. In order to investigate whether $\lambda_{\mathrm{SOT}, 4}^{100 \mathrm{meV}}$ and $\lambda_{\mathrm{SOT}, 3}^{100 \mathrm{meV}}$ simply describe the decay of secondary reflected spin current or whether they additionally exhibit a modification due to a potential position-dependence of the 
primary spin current, we divide the Pt layer into two regions: In the atomic layers Pt1 through Pt12 we switch off the mechanical force $-e \boldsymbol{E}$ that the electrons would otherwise experience due to the applied electric field $\boldsymbol{E}$. Only the atomic layers Pt13 through Co3 are subject to the mechanical force $-e \boldsymbol{E}$ in this modified calculation, which is based on Eq. (102). Thus, only Pt13 through Pt20 generate sizable SHE spin current (SHE in Co is small). The corresponding linear response coefficients are shown in Fig. 2 as circles for two values of broadening, $\Gamma=25 \mathrm{meV}$ and $\Gamma=100 \mathrm{meV}$. Switching off the mechanical force significantly perturbs the spin-current profile in the region Pt1 through Pt14 while from Pt15 onwards, the two spin-current profiles merge. Approaching the region with mechanical force switched off, i.e., approaching Pt12, the spin current (red circles in Fig. 2) in region $\mathrm{Pt} 13$ to $\mathrm{Pt} 17$ is suppressed according to

$$
q_{y x}^{\text {even }}(z)=\left[0.84-0.52 e^{-\left(z-z_{\mathrm{Pt} 12}\right) / \lambda_{\mathrm{SOT}, 5}^{100 \mathrm{meV}}}\right] e a_{0},
$$

where $\lambda_{\mathrm{SOT}, 5}^{100 \mathrm{meV}}=0.31 \mathrm{~nm}$. We find a slight $\Gamma$-dependence: $\lambda_{\mathrm{SOT}, 5}^{25 \mathrm{meV}}=0.28 \mathrm{~nm}$. In the region from Pt1 through Pt12 the spin current is well described by

$$
q_{y x}^{\text {even }}(z)=0.33 e^{\left(z-z_{\mathrm{Pt} 12}\right) / \lambda_{\mathrm{SOT}, 2 \mathrm{~T}}^{100 \mathrm{meV}}} e a_{0},
$$

with $\lambda_{\mathrm{SOT}, 2}^{100 \mathrm{meV}}=0.85 \mathrm{~nm}$. At $\Gamma=25 \mathrm{meV}$ the spincurrent profile in the region Pt1 through Pt14 cannot be described well by an exponential fit.

Comparing the lengths obtained from the exponential fits in Eq. (112), Eq. (113), Eq. (115) and Eq. (114) we find that $\lambda_{\mathrm{SOT}, 2}^{100 \mathrm{meV}}$ is substantially larger than the other three lengths: $\lambda_{\mathrm{SOT}, 2}^{100 \mathrm{meV}}>\lambda_{\mathrm{SOT}, 3}^{100 \mathrm{meV}} \approx \lambda_{\mathrm{SOT}, 4}^{100 \mathrm{meV}} \approx \lambda_{\mathrm{SOT}, 5}^{100 \mathrm{meV}}$. The length $\lambda_{\mathrm{SOT}, 2}^{100 \mathrm{meV}}$ describes the decay of spin current in $\mathrm{Pt}$ in a region of space where no spin current is generated (because the mechanical force is switched off in the region Pt1 through Pt12). This spin current, which is injected into the region Pt1-Pt12, originates only from the SHE in the region Pt13-Pt20. In contrast, the lengths $\lambda_{\mathrm{SOT}, 3}^{100 \mathrm{~m}}, \lambda_{\mathrm{SOT}, 4}^{100 \mathrm{meV}}$ and $\lambda_{\mathrm{SOT}, 5}^{100 \mathrm{mV}}$ describe the suppression of the total spin current close to interfaces and surfaces. The total spin current is the sum of spin current generated by SHE and spin current from the reflection at interfaces and surfaces. This reflected spin current is expected to be described by $\lambda_{\mathrm{SOT}, 2}^{100 \mathrm{meV}}$. Our finding that $\lambda_{\mathrm{SOT}, 3}^{100 \mathrm{meV}}, \lambda_{\mathrm{SOT}, 4}^{100 \mathrm{meV}}$ and $\lambda_{\mathrm{SOT}, 5}^{100 \mathrm{meV}}$ are all much smaller than $\lambda_{\mathrm{SOT}, 2}^{100 \mathrm{meV}}$ can only be explained if we assume that the primary spin current generated by SHE is itself modified close to surfaces and interfaces.

In Fig. 3 we show the torkance as a function of the region where the mechanical force is set to zero. If the mechanical force is switched off in all Pt layers and only active in the Co layers (data points at $L=\mathrm{Co} 1$ ), $t_{y x}^{\text {even }}$ is very small because the even torque arises dominantly from the SHE in Pt which is switched off when the mechanical force is set to zero. When the mechanical force

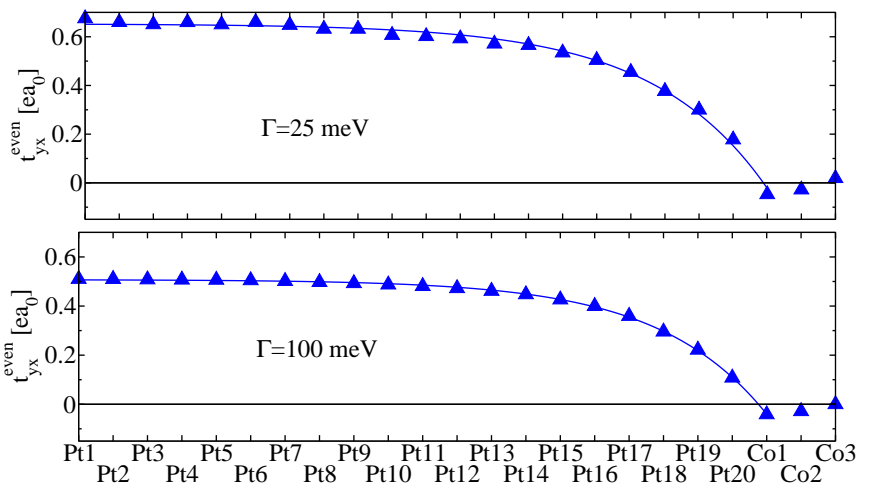

FIG. 3: Triangles: Torkances for broadenings of $\Gamma=25 \mathrm{meV}$ (upper graph) and $\Gamma=100 \mathrm{meV}$ (lower graph). For a given layer $L$ ( $L$ is specified on the horizontal axis), the mechanical force is switched off in the region from Pt1 through $L-1$ according to Eq. (102) and the resulting total torkance is shown by a blue triangle. Solid lines: Exponential fits according to Eq. (116).

is set to zero in the region from Pt1 through layer $L-1$, the torkance is well described by the fit

$$
t_{y x}^{\mathrm{even}}(z)=\left[0.65-0.68 e^{-\left(z_{\mathrm{Co} 1}-z\right) / \lambda_{\mathrm{SOT}, 1}^{25 \mathrm{meV}}}\right] e a_{0},
$$

where $\lambda_{\mathrm{SOT}, 1}^{25 \mathrm{meV}}=0.76 \mathrm{~nm}$. We find a weak $\Gamma$-dependence: $\lambda_{\mathrm{SOT}, 1}^{100 \mathrm{meV}}=0.71 \mathrm{~nm}$. At $\Gamma=100 \mathrm{meV}$ the spin current generated in a given atomic layer of $\mathrm{Pt}$ decays on the length scale of $\lambda_{\mathrm{SOT}, 2}^{100 \mathrm{meV}}$. Therefore, the SHE from layers $L$ that are further away from the Co layer than $\lambda_{\mathrm{SOT}, 2}^{100 \mathrm{meV}}$ cannot contribute to $t_{y x}^{\text {even }}$. Thus, we expect $\lambda_{\mathrm{SOT}, 1}^{100 \mathrm{meV}} \approx$ $\lambda_{\mathrm{SOT}, 2}^{100 \mathrm{meV}}$, which is indeed the case.

One main conclusion of this subsection is that for a sufficiently large broadening $\Gamma=100 \mathrm{meV}$ the $a b$ initio spin current profiles behave as expected from diffusive spin transport models. In particular, at $\Gamma=100 \mathrm{meV}$ the decay lengths of spin current extracted in various ways are found to be similar, namely $\lambda_{\mathrm{SOT}, 1}^{100 \mathrm{meV}}=0.71 \mathrm{~nm}$ and $\lambda_{\mathrm{SOT}, 2}^{100 \mathrm{meV}}=0.85 \mathrm{~nm}$. Similarly short but slightly longer length scales of roughly $1.5 \mathrm{~nm}$ have been observed in $\mathrm{Pt}$ in recent experiments [22, 38, 63, 64. A second conclusion from this subsection is that close to interfaces and surfaces the SHE conductivity is position-dependent. Therefore, close to interfaces and surfaces, the spin current profiles do not decay on the scale of $\lambda_{\mathrm{SOT}, 1}^{100 \mathrm{meV}} \approx$ $\lambda_{\mathrm{SOT}, 2}^{100 \mathrm{meV}}$ but instead significantly faster, namely according to $\lambda_{\mathrm{SOT}, 3}^{100 \mathrm{meV}} \approx \lambda_{\mathrm{SOT}, 4}^{100 \mathrm{meV}} \approx \lambda_{\mathrm{SOT}, 5}^{100 \mathrm{meV}} \approx 0.3 \mathrm{~nm}$.

\section{Even ISOT}

When the magnetization precesses in a circular orbit around the $z$ axis in the small-cone limit the current den- 


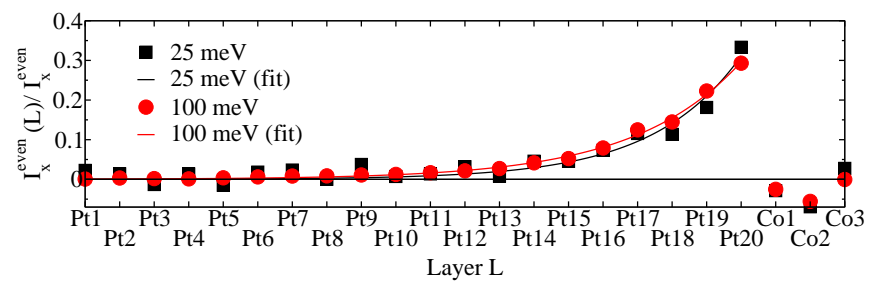

FIG. 4: Layer-resolved ISOT current $I_{x}^{\text {even }}(L)$ induced in $\mathrm{Co}(3) / \mathrm{Pt}(20)$ by magnetization dynamics. The total ISOT current is $I_{x}^{\text {even }}=\sum_{L} I_{x}^{\text {even }}(L)$. The relative contributions of the layers, i.e., $I_{x}^{\text {even }}(L) / I_{x}^{\text {even }}$, is shown for two values of broadening, $\Gamma=25 \mathrm{meV}$ and $\Gamma=100 \mathrm{meV}$. Solid lines: Exponential fit according to Eq. (118).

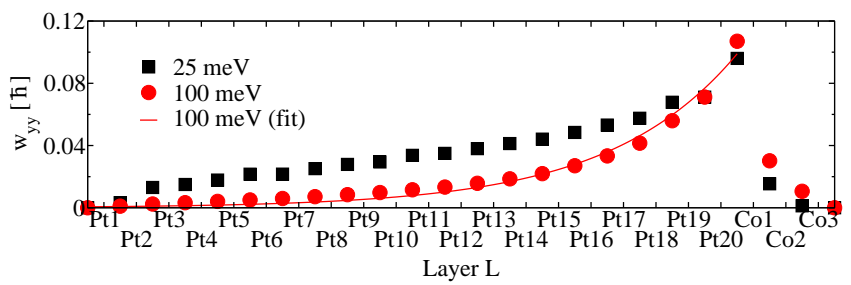

FIG. 5: Layer-resolved spin current induced by magnetization dynamics for two values of broadening, $\Gamma=25 \mathrm{meV}$ and $\Gamma=100 \mathrm{meV}$. The coefficient $w_{y y}$ describes spin current flowing in $z$ direction with spin pointing in $y$ direction and in phase with $I_{x}^{\text {even }}(t)$. Solid line: Fit according to Eq. (119).

sity

$$
\begin{aligned}
& \frac{J_{x, 25 \mathrm{meV}}^{\text {even }}(t)}{\omega}=-87 \frac{\mathrm{pAs}}{\mathrm{m}} \sin (\theta) \sin (\omega t), \\
& \frac{J_{x, 100 \mathrm{meV}}^{\text {even }}(t)}{\omega}=-68 \frac{\mathrm{pAs}}{\mathrm{m}} \sin (\theta) \sin (\omega t)
\end{aligned}
$$

is induced due to the even torkance $t_{y x}^{\text {even }}$ according to Eq. (68), where we used $A_{0}=t_{y x}^{\text {even }}$ and $A=23.8 a_{0}^{2}$.

As discussed in Sec. IIIB the ISOT current $I_{x}^{\text {even }}=$ $J_{x}^{\text {even }} L_{y}$ arises dominantly from the combination of spin pumping and ISHE. Since the spin current pumped into Pt decays, the layer-resolved ISOT current $I_{x}^{\text {even }}(L)$ is expected to reflect this spatial decay. Replacing $v_{\alpha}$ in Eq. (99) by $v_{\alpha}(L)$ (Eq. (100) yields the layer-resolved ISOT current $I_{x}^{\text {even }}(L)$ shown in Fig. 4. Inside the Pt layer, $I_{x}^{\text {even }}(L)$ is well described by an exponential function,

$$
I_{x}^{\text {even }}(z)=I_{x}^{\text {even }}\left(z_{\mathrm{Pt} 20}\right) e^{\left(z-z_{\mathrm{Pt} 20}\right) / \lambda_{\mathrm{ISOT}, 1}},
$$

where $z_{\mathrm{Pt} 20}$ is the $z$ coordinate of layer Pt20. Fitting Eq. (118) to the $I_{x}^{\text {even }}(L)$ profile obtained from first principles yields $\lambda_{\text {ISOT, } 1}^{25 \mathrm{meV}}=0.58 \mathrm{~nm}$ and $\lambda_{\mathrm{ISOT}, 1}^{100 \mathrm{meV}}=0.70 \mathrm{~nm}$.

In order to compare the spatial profile of the layerresolved ISOT current $I_{x}^{\text {even }}(L)$ with the spatial profile of the pumped spin current $Q_{y}(L, t)$ given by Eq. 105), we calculate the coefficients $w_{y y}(L)$, which are defined in Eq. (108). $w_{y y}(L)$ describes spin current in phase with
$I_{x}^{\text {even }}$ and with spin pointing in $y$ direction. Within $\mathrm{Pt}$, the $L$-dependence of $w_{y y}(L)$, shown in Fig. 5 , is approximately given by

$$
w_{y y}(z)=0.087 \hbar e^{\left(z-z_{\mathrm{Pt} 20}\right) / \lambda_{\mathrm{ISOT}, 2}^{100 \mathrm{meV}}},
$$

where $\lambda_{\mathrm{ISOT}, 2}^{100 \mathrm{meV}}=0.89 \mathrm{~nm}$. At smaller broadening $\Gamma=25 \mathrm{meV}$, the pumped spin current reaches the vacuum boundary at Pt1 and the resulting reflection of spin current needs to be considered according to Eq. 866). When $\lambda_{\mathrm{ISOT}, 2}^{25 \mathrm{meV}}$ is much larger than the thickness of Pt, the sinh function can be approximated:

$$
w_{y y}(z) \propto \frac{\sinh \frac{z-z_{\mathrm{P}+1}}{\lambda_{\mathrm{ISOT}, 2}^{25 \mathrm{~d}, 2}}}{\sinh \frac{z_{\mathrm{Pt} 20}-z_{\mathrm{Pt1}}}{\lambda_{\mathrm{ISOT}, 2}^{25 m e}}} \approx \frac{z-z_{\mathrm{Pt} 1}}{z_{\mathrm{Pt} 20}-z_{\mathrm{Pt} 1}},
$$

which explains the roughly linear profile of $w_{y y}(L)$ at $\Gamma=25 \mathrm{meV}$.

The ISOT currents shown in Fig. 4 decay faster in Pt than the spin currents in Fig. 5 Thus, Eq. (80), which predicts spin current and ISHE-current to be proportional, is violated, in particular at $\Gamma=25 \mathrm{meV}$. However, Eq. (80) is approximately satisfied at $\Gamma=100 \mathrm{meV}$, where both the ISOT current and the pumped spin current decay exponentially with $\lambda_{\mathrm{ISOT}, 1}^{100 \mathrm{meV}} \approx \lambda_{\mathrm{ISOT}, 2}^{100 \mathrm{meV}}$. The small difference $\lambda_{\mathrm{ISOT}, 2}^{100 \mathrm{meV}}-\lambda_{\mathrm{ISOT}, 1}^{100 \mathrm{meV}}=0.19 \mathrm{~nm}$ amounts to less than one $\mathrm{Pt}$ interlayer distance. Additionally, this spin current decay length $\lambda_{\mathrm{ISOT}, 1}^{100 \mathrm{meV}} \approx \lambda_{\mathrm{ISOT}, 2}^{100 \mathrm{meV}}$ is very similar to the one extracted in the previous subsection, i.e., $\lambda_{\mathrm{ISOT}, 1}^{100 \mathrm{meV}} \approx \lambda_{\mathrm{ISOT}, 2}^{100 \mathrm{meV}} \approx \lambda_{\mathrm{SOT}, 1}^{100 \mathrm{meV}} \approx \lambda_{\mathrm{SOT}, 2}^{100 \mathrm{meV}}$. This consistency between the various methods used to extract the spin current decay length implies that the model of Sec. IIIB provides a satisfactory description at sufficiently high broadening $\Gamma$.

In Eq. (79) the ISHE angle $\tan \gamma_{\text {ISHE }}$ is proportional to the quotient of ISOT current density and pumped spin current density. The different decay of ISOT current and pumped spin current described by $\lambda_{\mathrm{ISOT}, 1}^{100 \mathrm{meV}}$ and $\lambda_{\text {ISOT,2 }}^{100 \mathrm{meV}}$, respectively, therefore implies that $\tan \gamma_{\mathrm{ISHE}}$ is not constant but dependent on position. For large broadening we obtain $\tan \gamma_{\mathrm{ISHE}}^{100 \mathrm{meV}}(L=\mathrm{Pt} 20)=0.16$ and $\tan \gamma_{\mathrm{ISHE}}^{100 \mathrm{meV}}(L=\mathrm{Pt} 11)=0.077$, while for small broadening we obtain $\tan \gamma_{\mathrm{ISHE}}^{25 \mathrm{meV}}(L=\mathrm{Pt} 20)=0.27$ and $\tan \gamma_{\mathrm{ISHE}}^{25 \mathrm{meV}}(L=\mathrm{Pt} 11)=0.031$. Even for large broadening, the ISHE angle is significantly enhanced at the interface. The ISHE angles at the centre of $\mathrm{Pt}$, i.e., $\tan \gamma_{\mathrm{ISHE}}^{100 \mathrm{meV}}(L=\mathrm{Pt} 11)$ and $\tan \gamma_{\mathrm{ISHE}}^{25 \mathrm{meV}}(L=\mathrm{Pt} 11)$, are similar to the SHE angles determined in the previous section from the spin current in the center of Pt: $\tan \gamma_{\mathrm{SHE}}^{100 \mathrm{meV}}(L=\mathrm{Pt} 11)=0.109$ and $\tan \gamma_{\mathrm{SHE}}^{25 \mathrm{meV}}(L=$ $\mathrm{Pt} 11)=0.029$.

From Eq. (109) we obtain the spin-mixing conductance $\operatorname{Re} g_{25 \mathrm{meV}}^{\uparrow \downarrow}=1.8 \cdot 10^{19} \mathrm{~m}^{-2}$ and for $\Gamma=100 \mathrm{meV}$ a slightly larger value of $\operatorname{Re} g_{100 \mathrm{meV}}^{\uparrow \downarrow}=2.0 \cdot 10^{19} \mathrm{~m}^{-2}$. Eq. (84) 
provides an alternative way to extract the ISHE angle:

$$
\tan \bar{\gamma}_{\mathrm{ISHE}}^{100 \mathrm{meV}}=\frac{2 \pi t_{y x}^{\text {even }}}{e A \operatorname{Re} g_{100 \mathrm{meV}}^{\uparrow \downarrow} \lambda_{\mathrm{ISOT}, 2}^{100 \mathrm{meV}}}=0.15
$$

where the in-plane area of the unit cell is $A=23.8 a_{0}^{2}$ and the parameters $\lambda_{\mathrm{ISOT}, 2}^{100 \mathrm{meV}}=0.89 \mathrm{~nm}$ and $t_{y x, 100 \mathrm{meV}}^{\text {even }}=$ $0.53 \mathrm{ea}_{0}$ have been discussed above. In contrast to the layer-resolved ISHE angles, Eq. (121) describes an average over all those $\mathrm{Pt}$ layers that lie within the distance of $\lambda_{\mathrm{ISOT}, 2}^{100 \mathrm{meV}}$ from the Co layer. The result of $\tan \bar{\gamma}_{\mathrm{ISHE}}^{100 \mathrm{meV}}=$ 0.15 is very similar to the layer-resolved ISHE angle close to the interface of $\tan \gamma_{\mathrm{ISHE}}^{100 \mathrm{meV}}(L=\mathrm{Pt} 20)=0.16$.

Finally, we can also put Eq. (85) to a test using the parameters determined above:

$$
\lambda_{\mathrm{sd}}^{100 \mathrm{meV}}=\frac{\xi_{100 \mathrm{meV}} \hbar \pi \sigma_{x x}^{100 \mathrm{meV}}}{e^{2} \operatorname{Re} g_{100 \mathrm{meV}}^{\uparrow \downarrow}}=1.25 \mathrm{~nm} .
$$

While $\lambda_{\mathrm{sd}}^{100 \mathrm{meV}}$ is larger than $\lambda_{\mathrm{ISOT}, 2}^{100 \mathrm{meV}}$, the agreement between these two values is still satisfactory, corroborating the conclusion that the model of Sec. IIIB provides a satisfactory description for sufficiently large broadening. For small broadening Eq. (85) yields $\lambda_{\mathrm{sd}}^{25 \mathrm{meV}}=6.7 \mathrm{~nm}$, which is thicker than the Pt layer in our calculation and therefore justifies the linear approximation in Eq. (120).

\section{Odd SOT}

We obtain torkances per unit cell of $t_{x x, 25 \mathrm{meV}}^{\text {odd }}=$ $0.17 e a_{0}$ and $t_{x x, 100 \mathrm{meV}}^{\text {odd }}=0.15 e a_{0}$ at broadenings of $\Gamma=25 \mathrm{meV}$ and $\Gamma=100 \mathrm{meV}$, respectively. Dividing these torkances by the magnetic moment per unit cell of $\mu=5.78 \mu_{\mathrm{B}}$ we calculate the effective fields per applied electric field of $t_{x x, 25 \mathrm{meV}}^{\text {odd }} / \mu=0.0027 \mathrm{mTcm} / \mathrm{V}$ and $t_{x x, 100 \mathrm{meV}}^{\text {odd }} / \mu=0.0024 \mathrm{mTcm} / \mathrm{V}$.

In Fig. 6 we show the odd torkance as a function of the region with mechanical force switched off. If the mechanical force is switched off for Pt1 through Pt20 such that only the layers $\mathrm{Co} 1, \mathrm{Co} 2$ and $\mathrm{Co} 3$ are subject to it (see the data points at $L=\mathrm{Co} 1$ in the figure), the corresponding odd torque is not very different from the one with the mechanical force switched on everywhere (see the data points at $L=\mathrm{Pt} 1$ in the figure). If the mechanical force is applied only to layers Co2 and Co3 (see data points at $L=\mathrm{Co} 2$ in the figure) the resulting torkance is much smaller compared to the situation where all three Co layers are subject to it. Thus, the perturbation of the Co1 layer by the mechanical force is essential for the odd SOT in this system.

To produce a sizable odd torque in $\mathrm{Co}(3) / \mathrm{Pt}(20)$ it is therefore not crucial to switch on the mechanical force in the Pt layers but it suffices to apply this perturbation to the Co states. As a combined effect of broken inversion symmetry and SOI the spin of a

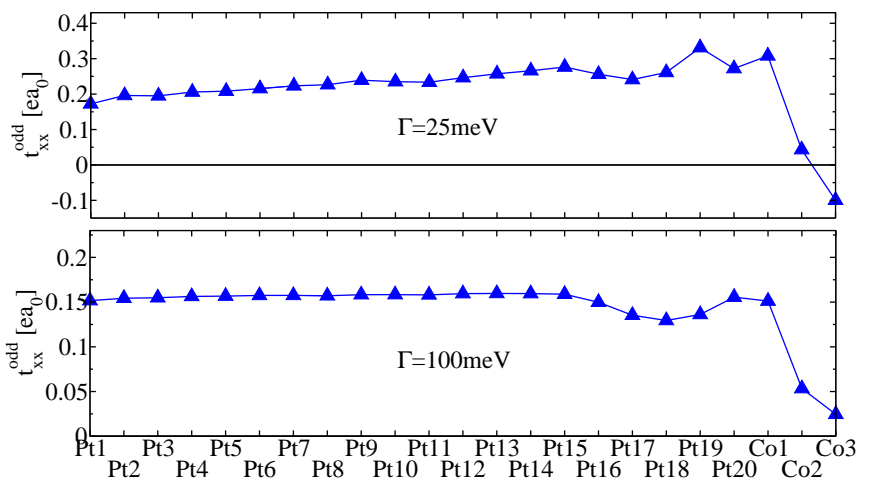

FIG. 6: Triangles: Torkances for broadenings of $\Gamma=25 \mathrm{meV}$ (upper graph) and $\Gamma=100 \mathrm{meV}$ (lower graph). For a given layer $L$ ( $L$ is specified on the horizontal axis), the mechanical force is switched off in the region from Pt1 through $L-1$ according to Eq. (102) and the resulting total torkance is shown by a blue triangle. Solid lines serve as guide to the eye.

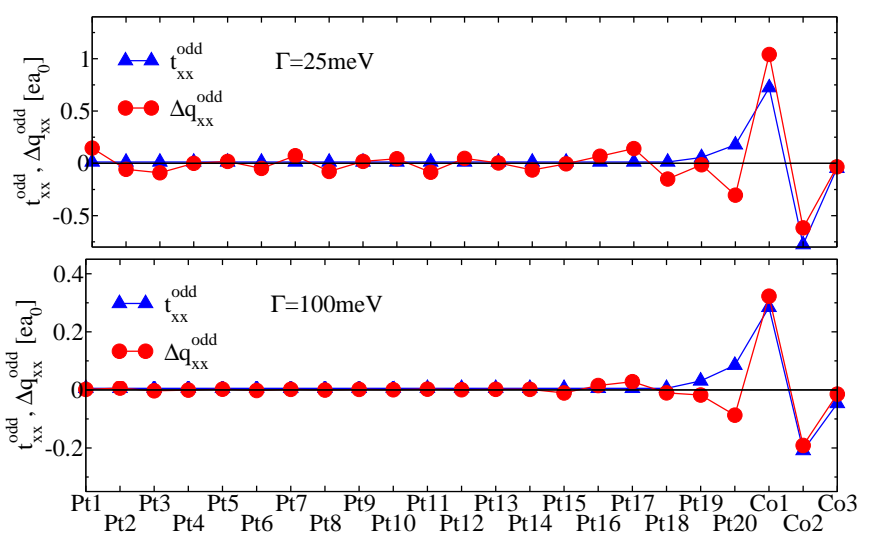

FIG. 7: Triangles: Layer-resolved odd torkance $t_{x x}^{\text {odd }}$ for broadenings of $\Gamma=25 \mathrm{meV}$ (upper graph) and $\Gamma=100 \mathrm{meV}$ (lower graph). Circles: Linear response coefficient of the layer-resolved spin flux $\Delta q_{x x}^{\text {odd }}$, Eq. (123). Solid lines serve as guide to the eye.

given wave function $\left|\Psi_{\boldsymbol{k} n}\right\rangle$ is correlated with the velocity $v_{k n n}$ [65]. As a result, the non-equilibrium spin density induced by an applied electric field combined with the exchange interaction gives rise to the odd component of the torkance [9, 10, 66]. Application of the mechanical force to Co, i.e., perturbation of the system via the velocity operator within the Co layer, produces therefore the dominant part of nonequilibrium spin density from which the odd torque arises in $\mathrm{Co}(3) / \mathrm{Pt}(20)$. This stands in marked contrast to the even torque in this system, which is mainly driven by SHE from Pt and thus very small if the mechanical force is turned off in all Pt layers, as shown in Fig. 3 ,

In Fig. (77) the layer-resolved odd torkance and the linear-response coefficient of spin flux into layer $L$, i.e.,

$$
\Delta q_{x x}^{\text {odd }}(L)=q_{x x}^{\text {odd }}(L)-q_{x x}^{\text {odd }}(L+1),
$$


are shown for two values of broadening, $\Gamma=25 \mathrm{meV}$ and $\Gamma=100 \mathrm{meV}$. For the layers Co1 through Co3 the layerresolved torkances coincide approximately with the spin fluxes like in the case of the even torque. This approximate agreement between odd spin fluxes and odd torques is not generally found in bilayer systems, for example they differ considerably in $\mathrm{O} / \mathrm{Co} / \mathrm{Pt}$ and $\mathrm{Al} / \mathrm{Co} / \mathrm{Pt}[31]$. For $\Gamma=100 \mathrm{meV}$ the magnetization of layer Pt20 experiences a torkance of $0.085 e a_{0}$. At the same time there is a spin flux out of layer Pt20 characterized by the coefficient $-\Delta q_{x x}^{\text {odd }}(L=\mathrm{Pt} 20)=0.087 e a_{0}$. This spin flux is transferred to the Co layer where it exerts a torque on the Co magnetization. The sum of torkance and spin flux coefficient of Pt20 amounts to $0.172 e a_{0}$ and approximately accounts for the total odd torkance of $0.15 e a_{0}$ at $\Gamma=100 \mathrm{meV}$. The angular momentum that gives rise to the odd torque on the magnetization is thus picked up from the lattice at Pt20 and roughly $50 \%$ of it is directly transferred to the magnetization of the Pt20 layer while the rest is transported to the Co layer via spin current. Above we have shown that the mechanical force on the Co1 layer is crucial to produce a sizable odd torque. Since the pick-up of angular momentum from the lattice by the spin system happens in Pt20, the hybridization of the Co1 states with the Pt20 states is thus essential.

\section{E. Odd ISOT}

According to Eq. (68) the current density

$$
\begin{aligned}
& \frac{J_{x, 25 \mathrm{meV}}^{\text {odd }}(t)}{\omega}=22 \frac{\mathrm{pAs}}{\mathrm{m}} \sin (\theta) \cos (\omega t), \\
& \frac{J_{x, 100 \mathrm{meV}}^{\text {odd }}(t)}{\omega}=19 \frac{\mathrm{pAs}}{\mathrm{m}} \sin (\theta) \cos (\omega t)
\end{aligned}
$$

is induced due to $t_{x x}^{\text {odd }}$ when the magnetization precesses around the $z$ axis in the small-cone limit. Here, we used $C_{0}=t_{x x}^{\text {odd }}$ and $A=23.8 a_{0}^{2}$. This contribution from $t_{x x}^{\text {odd }}$ is thus $-90^{\circ}$ phase shifted with respect to the contribution from $t_{y x}^{\text {even }}$ given in Eq. (117), i.e., it lags behind by a quarter period.

Since the mechanical force on the Co1 layer is crucial for the odd SOT according to Fig. 6 we expect that the odd ISOT current induced by magnetization dynamics flows mainly in the Co1 layer, because of the reciprocity between ISOT and SOT. This is indeed the case, as Fig. 8 shows. In particular, at $\Gamma=100 \mathrm{meV}$ the currents flowing in Co2, Co3 and the Pt layer are almost negligible. At the smaller broadening $\Gamma=25 \mathrm{meV}$ the induced ISOT currents in Co2, Co3 and $\mathrm{Pt}$ are larger, especially in the Co2 and Co3 layers, but the Co1 contribution to the ISOT current still strongly dominates.

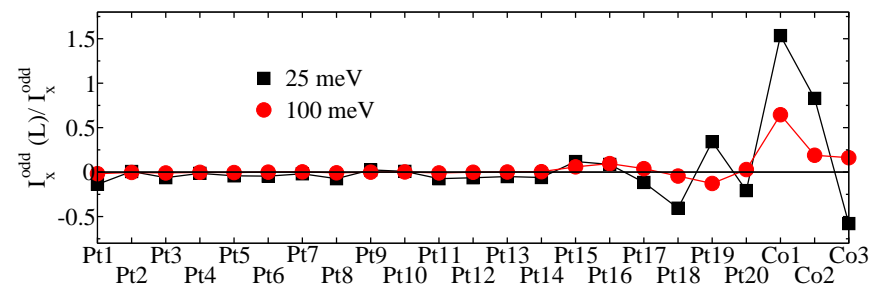

FIG. 8: Layer-resolved ISOT current $I_{x}^{\text {odd }}(L)$ induced in $\mathrm{Co}(3) / \mathrm{Pt}(20)$ by magnetization dynamics. The total ISOT current is $I_{x}^{\text {odd }}=\sum_{L} I_{x}^{\text {odd }}(L)$. The relative contributions of the layers, i.e., $I_{x}^{\text {odd }}(L) / I_{x}^{\text {odd }}$, is shown for two values of broadening, $\Gamma=25 \mathrm{meV}$ (squares) and $\Gamma=100 \mathrm{meV}$ (circles). Solid lines serve as guide to the eye.

\section{SUMMARY}

SOT and ISOT are reciprocal effects. Both of them can be expressed conveniently in terms of the torkance tensor $\boldsymbol{t}(\hat{\boldsymbol{M}})$, which depends on the magnetization direction $\hat{M}$. In the case of the SOT phenomenon, the torque $\boldsymbol{T}(\hat{\boldsymbol{M}})$ on the magnetization due to the application of an electric field $\boldsymbol{E}$ is given by $\boldsymbol{T}(\hat{\boldsymbol{M}})=\boldsymbol{t}(\hat{\boldsymbol{M}}) \boldsymbol{E}$. If $\hat{\boldsymbol{M}}$ changes as a function of time, the reciprocal effect, the ISOT, can be observed. It consists in the generation of a current density $\boldsymbol{j}(t)=[\boldsymbol{t}(-\hat{\boldsymbol{M}}(t))]^{\mathrm{T}}\left[\hat{\boldsymbol{M}}(t) \times \frac{d \hat{\boldsymbol{M}}(t)}{d t}\right] / V$, where $V$ is the unit cell volume. Magentization dynamics driven effects, such as ISOT and Gilbert damping, can be consistently derived in time-dependent perturbation theory using a time-dependent exchange field. The same expressions are obtained by rewriting general many body susceptibilities in terms of the Kohn-Sham susceptibilities. On the basis of the SOT-ISOT reciprocity relations and recent experimental results for the SOT in bilayer systems, we predict the angular dependence of the FMR-driven ISOT in bilayers. We find that measurements of the dc voltage associated with the FMRdriven ISOT are insufficient to determine $\boldsymbol{t}(\hat{\boldsymbol{M}})$ in general and that additionally the ac voltage needs to be measured phase-sensitively to determine $\boldsymbol{t}(\hat{\boldsymbol{M}})$ completely. Within the Kubo linear response formalism we investigate SOTs and ISOTs in $\mathrm{Co} / \mathrm{Pt}(111)$ magnetic bilayers using the electronic structure provided from first principles density functional theory. Magnetization-dynamics induced charge currents and spin currents are resolved on the atomic scale to extract model parameters and to expose the mechanisms underlying the ISOT. Likewise the spin currents accompanying the SOT are resolved on the atomic scale for the same purposes. It is found that SHE and ISHE are modified close to interfaces and surfaces. Comparison of the various currents accompanying SOT on the one hand and ISOT on the other hand highlights the reciprocity of the two phenomena on the microscopic scale.

We gratefully acknowledge computing time on the su- 
percomputers JUQUEEN and JUROPA at Jülich Supercomputing Center and funding under the HGF-YIG programme VH-NG-513.

\section{Appendix A: Magnetocrystalline anisotropy and the static torque-torque correlation function} by

The torque due to the field $\boldsymbol{H}^{\mathrm{MAE}}$, Eq. (30), is given

$$
\begin{aligned}
\delta \boldsymbol{T}^{\mathrm{MAE}} & =\mu_{0} M V \hat{\boldsymbol{M}} \times \boldsymbol{H}^{\mathrm{MAE}} \\
& =-M V\left[\bar{\Omega}^{\mathrm{xc}}+\frac{G_{\mathcal{T}}^{\mathrm{R}}(\hbar \omega=0, \hat{\boldsymbol{M}})}{M V \hbar}\right] \hat{\boldsymbol{M}} \times \delta \hat{\boldsymbol{M}}
\end{aligned}
$$

where we used Eq. (48) to express $\chi$ in terms of the torque-torque correlation function $G_{\mathcal{T}}^{\mathrm{R}}$. Eq. (A1) can be related easily to anisotropy constants. For example, in the case of uniaxial anisotropy, i.e., $E(\theta)=V K_{1} \sin ^{2} \theta$, one obtains

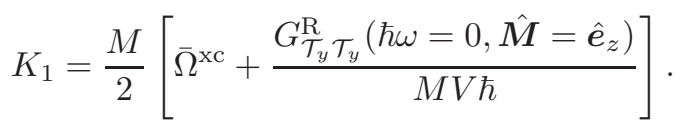

In the following we show that Eq. (A1), which was obtained within the many-electron response formalism of Sec. IIC, can also be obtained directly from the torque exerted on the magnetization by the Kohn-Sham electrons. Denoting the Kohn-Sham wavefunctions by $\left|\psi_{\boldsymbol{k} n}\right\rangle$ and the occupancies by $f_{\boldsymbol{k} n}$ we can write

$$
\begin{aligned}
\delta \boldsymbol{T}^{\mathrm{MAE}}= & -\delta\left\{\frac{1}{\mathcal{N}} \sum_{\boldsymbol{k} n} f_{\boldsymbol{k} n}\left\langle\psi_{\boldsymbol{k} n}|\mathcal{T}| \psi_{\boldsymbol{k} n}\right\rangle\right\} \\
= & -\frac{1}{\mathcal{N}} \sum_{\boldsymbol{k} n} f_{\boldsymbol{k} n}\left\langle\psi_{\boldsymbol{k} n}|\delta \mathcal{T}| \psi_{\boldsymbol{k} n}\right\rangle \\
& -\frac{1}{\mathcal{N}} \sum_{\boldsymbol{k} n} \delta f_{\boldsymbol{k} n}\left\langle\psi_{\boldsymbol{k} n}|\mathcal{T}| \psi_{\boldsymbol{k} n}\right\rangle \\
& -2 \operatorname{Re} \frac{1}{\mathcal{N}} \sum_{\boldsymbol{k} n} f_{\boldsymbol{k} n}\left\langle\psi_{\boldsymbol{k} n}|\mathcal{T} \delta| \psi_{\boldsymbol{k} n}\right\rangle .
\end{aligned}
$$

From $\delta \mathcal{T}=\boldsymbol{m} \times \delta \hat{M} \Omega^{\mathrm{xc}}$ we obtain for the first term

$$
-\frac{1}{\mathcal{N}} \sum_{\boldsymbol{k} n} f_{\boldsymbol{k} n}\left\langle\psi_{\boldsymbol{k} n}|\delta \mathcal{T}| \psi_{\boldsymbol{k} n}\right\rangle=-M V \bar{\Omega}^{\mathrm{xc}} \hat{\boldsymbol{M}} \times \delta \hat{\boldsymbol{M}}
$$

Using for the remaining terms

$$
\delta\left|\psi_{\boldsymbol{k} n}\right\rangle=\sum_{m \neq n} \frac{\left|\psi_{\boldsymbol{k} m}\right\rangle\left\langle\psi_{\boldsymbol{k} m}|\mathcal{T}| \psi_{\boldsymbol{k} n}\right\rangle}{\mathcal{E}_{\boldsymbol{k} n}-\mathcal{E}_{\boldsymbol{k} m}} \cdot(\hat{\boldsymbol{M}} \times \delta \hat{\boldsymbol{M}})
$$

and

$$
\delta f_{\boldsymbol{k} n}=-\delta\left(\mathcal{E}_{\mathrm{F}}-\mathcal{E}_{\boldsymbol{k} n}\right)\left\langle\psi_{\boldsymbol{k} n}|\mathcal{T}| \psi_{\boldsymbol{k} n}\right\rangle \cdot(\hat{\boldsymbol{M}} \times \delta \hat{\boldsymbol{M}})
$$

and

$$
\begin{gathered}
G_{\mathcal{T}_{\alpha} \mathcal{T}_{\beta}}^{\mathrm{R}}=\frac{2 \hbar}{\mathcal{N}} \sum_{\boldsymbol{k} n} \sum_{m \neq n} f_{\boldsymbol{k} n} \operatorname{Re} \frac{\left\langle\psi_{\boldsymbol{k} n}\left|\mathcal{T}_{\alpha}\right| \psi_{\boldsymbol{k} m}\right\rangle\left\langle\psi_{\boldsymbol{k} m}\left|\mathcal{T}_{\beta}\right| \psi_{\boldsymbol{k} n}\right\rangle}{\mathcal{E}_{\boldsymbol{k} n}-\mathcal{E}_{\boldsymbol{k} m}} \\
-\frac{\hbar}{\mathcal{N}} \sum_{\boldsymbol{k} n} \delta\left(\mathcal{E}_{\mathrm{F}}-\mathcal{E}_{\boldsymbol{k} n}\right)\left\langle\psi_{\boldsymbol{k} n}\left|\mathcal{T}_{\alpha}\right| \psi_{\boldsymbol{k} n}\right\rangle\left\langle\psi_{\boldsymbol{k} n}\left|\mathcal{T}_{\beta}\right| \psi_{\boldsymbol{k} n}\right\rangle
\end{gathered}
$$

one can easily show that Eq. (A1) and Eq. (A3) agree.

The Kohn-Sham Hamiltonian can be decomposed as

$$
H(\boldsymbol{r})=H_{\mathrm{KIN}}+V(\boldsymbol{r})-\boldsymbol{m} \cdot \hat{\boldsymbol{M}} \Omega^{\mathrm{xc}}(\boldsymbol{r})+H_{\mathrm{SOI}},
$$

where $H_{\mathrm{KIN}}$ describes the kinetic energy, $V(\boldsymbol{r})$ is the spinindependent part of the effective potential and $H_{\mathrm{SOI}}$ describes the spin-orbit interaction. Using $\left[H_{\mathrm{KIN}}, \sigma_{\beta}\right]=0$, $\left[V(\boldsymbol{r}), \sigma_{\beta}\right]=0$ and $\left[\sigma_{\alpha}, \sigma_{\beta}\right]=2 i \epsilon_{\alpha \beta \gamma} \sigma_{\gamma}$ one can show the following identity for the torque operator:

$$
\mathcal{T}_{\beta}=\frac{i}{2}\left[H-H_{\mathrm{SOI}}, \sigma_{\beta}\right]
$$

Substituting $\mathcal{T}_{\beta}$ in Eq. (A7) by Eq. A9 and inserting the resulting expression for $G_{\mathcal{T}_{\alpha}}^{\mathrm{R}} \mathcal{T}_{\beta}$ into Eq. A1 we obtain

$$
\begin{aligned}
& \delta \boldsymbol{T}^{\mathrm{MAE}}=-\frac{1}{\mathcal{N}} \sum_{\boldsymbol{k} n \beta}(\hat{\boldsymbol{M}} \times \delta \hat{\boldsymbol{M}})_{\beta}\{ \\
& f_{\boldsymbol{k} n} \operatorname{Im} \sum_{m \neq n} \frac{\left\langle\psi_{\boldsymbol{k} n}|\mathcal{T}| \psi_{\boldsymbol{k} m}\right\rangle\left\langle\psi_{\boldsymbol{k} m}\left|\left[H_{\mathrm{SOI}}, \sigma_{\beta}\right]\right| \psi_{\boldsymbol{k} n}\right\rangle}{\mathcal{E}_{\boldsymbol{k} n}-\mathcal{E}_{\boldsymbol{k} m}}+ \\
& \left.+\frac{i}{2} \delta\left(\mathcal{E}_{\mathrm{F}}-\mathcal{E}_{\boldsymbol{k} n}\right)\left\langle\psi_{\boldsymbol{k} n}|\mathcal{T}| \psi_{\boldsymbol{k} n}\right\rangle\left\langle\psi_{\boldsymbol{k} n}\left|\left[H_{\mathrm{SOI}}, \sigma_{\beta}\right]\right| \psi_{\boldsymbol{k} n}\right\rangle\right\} .
\end{aligned}
$$

Eq. A10 is well-suited for the calculation of the magnetocrystalline anisotropy within Kohn-Sham densityfunctional-theory codes. In contrast, the direct application of Eq. A1 in practice would suffer from the following disadvantage: Since the magnetocrystalline anisotropy energy is usually much smaller than the average exchange field $\bar{\Omega}^{\mathrm{xc}}$, one would need to calculate both $\bar{\Omega}^{\mathrm{xc}}$ as well as the torque-torque correlation function $G_{\mathcal{T}_{\alpha}}^{\mathrm{R}} \mathcal{T}_{\beta}$ with very high precision if one wanted to use directly Eq. A1 for the determination of the magnetocrystalline anisotropy.

In the absence of SOI we have $H_{\mathrm{SOI}}=0$ and Eq. A9 simplifies to $\mathcal{T}_{\beta}=i\left[H, \sigma_{\beta}\right] / 2$. Since $\left|\psi_{\boldsymbol{k} n}\right\rangle$ is an eigenstate of $H$ it follows that $\left\langle\psi_{\boldsymbol{k} n}\left|\mathcal{T}_{\beta}\right| \psi_{\boldsymbol{k} n}\right\rangle=0$ and therefore the last term in Eq. (A7) vanishes. Thus, in the absence of SOI Eq. A7 can be written as

$$
G_{\mathcal{T}_{\alpha}}^{\mathrm{R}} \mathcal{T}_{\beta}=\frac{\hbar}{\mathcal{N}} \sum_{\boldsymbol{k} n} f_{\boldsymbol{k} n} \operatorname{Im}\left\langle\psi_{\boldsymbol{k} n}\left|\mathcal{T}_{\alpha} \sigma_{\beta}\right| \psi_{\boldsymbol{k} n}\right\rangle .
$$

Using $\sigma_{\alpha} \sigma_{\beta}=\delta_{\alpha \beta}+i \epsilon_{\alpha \beta \gamma} \sigma_{\gamma}$ one can derive Eq. (44) from Eq. (A11.

\footnotetext{
* Corresp. author: f.freimuth@fz-juelich.de
} 
[1] L. Berger, Phys. Rev. B 33, 1572 (1986).

[2] G. E. Volovik, Journal of Physics C: Solid State Physics 20, L83 (1987).

[3] S. E. Barnes and S. Maekawa, Phys. Rev. Lett. 98, 246601 (2007).

[4] K.-J. Lee, M. D. Stiles, H.-W. Lee, J.-H. Moon, K.-W. Kim, and S.-W. Lee, Physics reports 531, 89 (2013).

[5] S. A. Yang, G. S. D. Beach, C. Knutson, D. Xiao, Q. Niu, M. Tsoi, and J. L. Erskine, Phys. Rev. Lett. 102, 067201 (2009).

[6] T. Schulz, R. Ritz, A. Bauer, M. Halder, M. Wagner, C. Franz, C. Pfleiderer, K. Everschor, M. Garst, and A. Rosch, Nature physics 8, 301 (2012).

[7] K.-W. Kim, J.-H. Moon, K.-J. Lee, and H.-W. Lee, Phys. Rev. Lett. 108, 217202 (2012).

[8] G. Tatara, N. Nakabayashi, and K.-J. Lee, Phys. Rev. B 87, 054403 (2013).

[9] I. Garate and A. H. MacDonald, Phys. Rev. B 80, 134403 (2009).

[10] A. Manchon and S. Zhang, Phys. Rev. B 79, 094422 (2009).

[11] D. A. Pesin and A. H. MacDonald, Phys. Rev. B 86, 014416 (2012).

[12] E. van der Bijl and R. A. Duine, Phys. Rev. B 86, 094406 (2012).

[13] X. Wang and A. Manchon, Phys. Rev. Lett. 108, 117201 (2012).

[14] P. M. Haney, H.-W. Lee, K.-J. Lee, A. Manchon, and M. D. Stiles, Phys. Rev. B 87, 174411 (2013).

[15] P. M. Haney, H.-W. Lee, K.-J. Lee, A. Manchon, and M. D. Stiles, Phys. Rev. B 88, 214417 (2013).

[16] K. M. D. Hals and A. Brataas, Phys. Rev. B 88, 085423 (2013).

[17] K.-W. Kim, S.-M. Seo, J. Ryu, K.-J. Lee, and H.-W. Lee, Phys. Rev. B 85, 180404 (2012).

[18] C. Ciccarelli, K. M. D. Hals, A. Irvine, V. Novak, Y. Tserkovnyak, H. Kurebayashi, A. Brataas, and A. Ferguson, Nature nanotechnology 10, 50 (2014).

[19] K. M. D. Hals and A. Brataas, Phys. Rev. B 91, 214401 (2015).

[20] I. Mihai Miron, G. Gaudin, S. Auffret, B. Rodmacq, A. Schuhl, S. Pizzini, J. Vogel, and P. Gambardella, Nature Mater. 9, 230 (2010).

[21] I. Mihai Miron, K. Garello, G. Gaudin, P.-J. Zermatten, M. V. Costache, S. Auffret, S. Bandiera, B. Rodmacq, A. Schuhl, and P. Gambardella, Nature 476, 189 (2011).

[22] L. Liu, O. J. Lee, T. J. Gudmundsen, D. C. Ralph, and R. A. Buhrman, Phys. Rev. Lett. 109, 096602 (2012).

[23] L. Liu, C.-F. Pai, Y. Li, H. W. Tseng, D. C. Ralph, and R. A. Buhrman, Science 336, 555 (2012).

[24] K. Garello, I. M. Miron, C. O. Avci, F. Freimuth, Y. Mokrousov, S. Blügel, S. Auffret, O. Boulle, G. Gaudin, and P. Gambardella, Nature Nanotech. 8, 587 (2013).

[25] J. Kim, J. Sinha, M. Hayashi, M. Yamanouchi, S. Fukami, T. Suzuki, S. Mitani, and H. Ohno, Nature Mater. 12, 240 (2013).

[26] X. Qiu, P. Deorani, K. Narayanapillai, K.-S. Lee, K.-J. Lee, H.-W. Lee, and H. Yang, Scientific reports 4 (2014).

[27] C.-F. Pai, L. Liu, Y. Li, H. W. Tseng, D. C. Ralph, and R. A. Buhrman, Appl. Phys. Lett. 101, 122404 (2012).

[28] P. P. J. Haazen, E. Mure, J. H. Franken, R. Lavrijsen, H. J. M. Swagten, and B. Koopmans, Nature Mater. 12, 299 (2013).
[29] L. Thomas, K. Ryu, S. Yang, and S. S. P. Parkin, Nature Nanotech. 8, 527 (2013).

[30] S. Emori, U. Bauer, S. Ahn, E. Martinez, and G. S. D. Beach, Nature Mater. 12, 611 (2013).

[31] F. Freimuth, S. Blügel, and Y. Mokrousov, Phys. Rev. B 90, 174423 (2014).

[32] R. Urban, G. Woltersdorf, and B. Heinrich, Phys. Rev. Lett. 87, 217204 (2001).

[33] Y. Tserkovnyak, A. Brataas, and G. E. W. Bauer, Phys. Rev. Lett. 88, 117601 (2002).

[34] O. Mosendz, J. E. Pearson, F. Y. Fradin, G. E. W. Bauer, S. D. Bader, and A. Hoffmann, Phys. Rev. Lett. 104, 046601 (2010).

[35] O. Mosendz, V. Vlaminck, J. E. Pearson, F. Y. Fradin, G. E. W. Bauer, S. D. Bader, and A. Hoffmann, Phys. Rev. B 82, 214403 (2010).

[36] F. D. Czeschka, L. Dreher, M. S. Brandt, M. Weiler, M. Althammer, I.-M. Imort, G. Reiss, A. Thomas, W. Schoch, W. Limmer, et al., Phys. Rev. Lett. 107, 046601 (2011).

[37] A. Azevedo, L. H. Vilela-Leao, R. L. Rodriguez-Suarez, A. F. Lacerda Santos, and S. M. Rezende, Phys. Rev. B 83, 144402 (2011).

[38] M. Weiler, M. Althammer, M. Schreier, J. Lotze, M. Pernpeintner, S. Meyer, H. Huebl, R. Gross, A. Kamra, J. Xiao, et al., Phys. Rev. Lett. 111, 176601 (2013).

[39] H. Jiao and G. E. W. Bauer, Phys. Rev. Lett. 110, 217602 (2013).

[40] D. Wei, M. Obstbaum, M. Ribow, C. H. Back, and G. Woltersdorf, Nature Communications 5 (2014).

[41] M. Weiler, J. M. Shaw, H. T. Nembach, and T. J. Silva, Phys. Rev. Lett. 113, 157204 (2014).

[42] C. Hahn, G. de Loubens, M. Viret, O. Klein, V. V. Naletov, and J. Ben Youssef, Phys. Rev. Lett. 111, 217204 (2013).

[43] R. A. Duine, Phys. Rev. B 79, 014407 (2009).

[44] Y. Tserkovnyak and S. A. Bender, Phys. Rev. B 90, 014428 (2014).

[45] F. Freimuth, S. Blügel, and Y. Mokrousov, Journal of physics: Condensed matter 26, 104202 (2014).

[46] F. Freimuth, R. Bamler, Y. Mokrousov, and A. Rosch, Phys. Rev. B 88, 214409 (2013).

[47] H. Ebert, S. Mankovsky, D. Ködderitzsch, and P. J. Kelly, Phys. Rev. Lett. 107, 066603 (2011).

[48] Z. Qian and G. Vignale, Phys. Rev. Lett. 88, 056404 (2002).

[49] R. R. Birss, Symmetry and Magnetism (North-Holland, Amsterdam, 1964).

[50] I. Garate and A. MacDonald, Phys. Rev. B 79, 064403 (2009).

[51] K. Ando, T. Yoshino, and E. Saitoh, Applied Physics Letters 94, 152509 (2009).

[52] M. Gradhand, D. V. Fedorov, P. Zahn, I. Mertig, Y. Otani, Y. Niimi, L. Vila, and A. Fert, SPIN 02, 1250010 (2012).

[53] W. Zhang, M. B. Jungfleisch, W. Jiang, Y. Liu, J. E. Pearson, S. G. E. t. Velthuis, A. Hoffmann, F. Freimuth, and Y. Mokrousov, Phys. Rev. B 91, 115316 (2015).

[54] A. Vedyayev, N. Ryzhanova, N. Strelkov, and B. Dieny, Phys. Rev. Lett. 110, 247204 (2013).

[55] T. Nan, S. Emori, C. T. Boone, X. Wang, T. M. Oxholm, J. G. Jones, B. M. Howe, G. J. Brown, and N. X. Sun, Phys. Rev. B 91, 214416 (2015). 
[56] W. Zhang, W. Han, X. Jiang, S.-H. Yang, and S. S. P. Parkin, Nature physics 11, 496 (2015).

[57] G. Géranton, F. Freimuth, S. Blügel, and Y. Mokrousov, Phys. Rev. B 91, 014417 (2015).

[58] K. Gilmore, Y. U. Idzerda, and M. D. Stiles, Phys. Rev. Lett. 99, 027204 (2007).

[59] J. Kuneš and V. Kamberský, Phys. Rev. B 65, 212411 (2002).

[60] F. Freimuth, Y. Mokrousov, D. Wortmann, S. Heinze, and S. Blügel, Phys. Rev. B 78, 035120 (2008).

[61] A. A. Mostofi, J. R. Yates, Y.-S. Lee, I. Souza, D. Vanderbilt, and N. Marzari, Computer Physics Communications 178, 685 (2008).

[62] N. Marzari, A. A. Mostofi, J. R. Yates, I. Souza, and
D. Vanderbilt, Rev. Mod. Phys. 84, 1419 (2012).

[63] M. Althammer, S. Meyer, H. Nakayama, M. Schreier, S. Altmannshofer, M. Weiler, H. Huebl, S. Geprägs, M. Opel, R. Gross, et al., Phys. Rev. B 87, 224401 (2013).

[64] W. Zhang, V. Vlaminck, J. E. Pearson, R. Divan, S. D. Bader, and A. Hoffmann, Applied physics letters 103, 242414 (2013).

[65] V. Edelstein, Solid State Communications 73, 233 (1990).

[66] A. Chernyshov, M. Overby, X. Liu, J. K. Furdyna, Y. Lyanda-Geller, and L. P. Rokhinson, Nature Phys. 5, 656 (2009). 